\title{
INFINITE-STEP NILSYSTEMS, INDEPENDENCE AND COMPLEXITY
}

\author{
PANDENG DONG, SEBASTIÁN DONOSO, ALEJANDRO MAASS, SONG SHAO, \\ AND XIANGDONG YE
}

\begin{abstract}
An $\infty$-step nilsystem is an inverse limit of minimal nilsystems. In this article is shown that a minimal distal system is an $\infty$-step nilsystem if and only if it has no nontrivial pairs with arbitrarily long finite IP-independence sets. Moreover, it is proved that any minimal system without nontrivial pairs with arbitrarily long finite IP-independence sets is an almost one to one extension of its maximal $\infty$-step nilfactor, and each invariant ergodic measure is isomorphic (in the measurable sense) to the Haar measure on some $\infty$-step nilsystem. The question if such a system is uniquely ergodic remains open. In addition, the topological complexity of an $\infty$-step nilsystem is computed, showing that it is polynomial for each nontrivial open cover.
\end{abstract}

\section{INTRODUCTION}

In this paper we introduce the notion of $\infty$-step nilsystem and study its relationship with the concept of independence. We also study its topological complexity. In this section, first we discuss the motivations for this subject and then we state the main results of the article.

1.1. Motivations. By a topological dynamical system (t.d.s. for short) we mean a pair $(X, T)$, where $X$ is a compact metric space and $T: X \rightarrow X$ is a homeomorphism.

There are several motivations for studying this subject. The first one comes from the so called local entropy theory, for a survey see [12]. Each t.d.s. admits a maximal zero topological entropy factor, and this factor is induced by the smallest closed invariant equivalence relation containing entropy pairs [5]. In [22], entropy pairs are characterized as those pairs that admit an interpolating set of positive density. Later on, the notions of sequence entropy pairs [20] and untame pairs (called scrambled pairs in [17]) were introduced. In [24] the concept of independence was extensively studied and used to unify the afore mentioned notions. Let $(X, T)$ be a t.d.s. and $\mathcal{A}=\left(A_{1}, \ldots, A_{k}\right)$ be a tuple of subsets of $X$. We say that a subset $F \subseteq \mathbb{Z}_{+}$is an independence set for $\mathcal{A}$ if for any nonempty finite subset $J \subseteq F$ and any $s=(s(j): j \in J) \in\{1, \ldots, k\}^{J}$ we have $\bigcap_{j \in J} T^{-j} A_{s(j)} \neq \emptyset$. It is shown that a

P. Dong and X. Ye were supported by NNSF of China (11071231). S. Donoso and A. Maass were supported by Basal-CMM and FONDAP-CRG grants. S. Shao was supported by NNSF of China (10871186) and Program for New Century Excellent Talents in University. 
pair of points $x, y$ in $X$ is a sequence entropy pair if and only if each $\mathcal{A}=\left(A_{1}, A_{2}\right)$, with $A_{1}$ and $A_{2}$ neighborhoods of $x$ and $y$ respectively, has arbitrarily long finite independence sets. Also, the pair is an untame pair if and only if each $\mathcal{A}=\left(A_{1}, A_{2}\right)$ as before has infinite independence sets. It is known that each t.d.s. admits a maximal zero sequence entropy factor, i.e. a null factor [20, which is induced by the smallest closed invariant equivalence relation containing sequence entropy pairs, and a maximal tame factor [24], which is induced by the smallest closed invariant equivalence relation containing untame pairs. It was shown $([20,24,10])$ that a minimal null (resp. tame) system is an almost 1-1 extension of an equicontinuous t.d.s. and is uniquely ergodic. For a similar study see [27]. Moreover, in the equicontinuous case the uniquely ergodic measure is measure theoretical isomorphic to the Haar measure of the underlying Abelian group.

To get a better understanding of the role of the notion of independence in t.d.s., in [18, 19] the authors systematically investigate the independence for a given collection of subsets of $\mathbb{Z}_{+}$. For a finite subset $\left\{p_{1}, \ldots, p_{m}\right\}$ of $\mathbb{N}$, the finite IP-set generated by $\left\{p_{1}, \ldots, p_{m}\right\}$ is the set $\left\{\epsilon_{1} p_{1}+\ldots+\epsilon_{m} p_{m}: \epsilon_{i} \in\{0,1\}, 1 \leq i \leq m\right\} \backslash\{0\}$. The notion of Ind $_{\text {fip }}$-pair is introduced and studied in [19]: a pair of points $(x, y)$ in $X$ is an Ind $_{\text {fip }}$-pair if and only if each $\mathcal{A}=\left(A_{1}, A_{2}\right)$, with $A_{1}$ and $A_{2}$ neighborhoods of $x$ and $y$ respectively, has arbitrarily long finite IP-independence sets. Among other results it is shown that the $\operatorname{Ind}_{f i p}$-pair relation has the lifting property, i.e. if $\pi:(X, T) \longrightarrow$ $(Y, S)$ is a factor map between two t.d.s. then $\pi \times \pi\left(\operatorname{Ind}_{\text {fip }}(X, T)\right)=\operatorname{Ind}_{\text {fip }}(Y, S)$, where $\operatorname{Ind}_{f i p}(X, T)$ is the set of all $\operatorname{Ind}_{f i p}$-pairs of $(X, T)$. It is clear that,

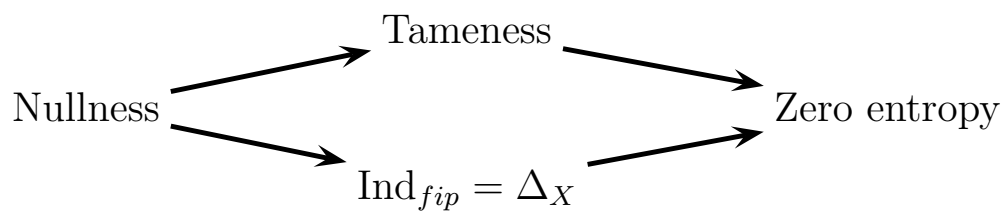

So it is interesting to understand the dynamical properties of a minimal t.d.s. without Ind fip $_{\text {-pairs. }}$

A second motivation comes from the study of the dynamics of nilsystems. Let $(X, \mathcal{B}, \mu, T)$ be an ergodic system.

In [15, to study the convergence of some non-conventional ergodic averages in this system, the authors proved that the characteristic factors for such averages in $L^{2}(X, \mathcal{B}, \mu)$ are $d$-step nilsystems for some integer $d \geq 1$ (see also [34]). Then, in the topological setting, in [16] the authors defined the notion of regionally proximal relation of order $d$ associated to a t.d.s. $(X, T), \mathbf{R} \mathbf{P}^{[d]}$, and showed that if the system is minimal and distal then $\mathbf{R} \mathbf{P}^{[d]}$ is an equivalence relation and $\left(X / \mathbf{R} \mathbf{P}^{[d]}, T\right)$ is the maximal $d$-step nilfactor of the system. In a recent preprint [29], this result was generalized to arbitrarily minimal t.d.s. When studying minimal distal systems carefully one finds that if $(x, y) \in \mathbf{R} \mathbf{P}^{[d]}$ for some integer $d$ then each $\mathcal{A}=\left(A_{1}, A_{2}\right)$, with $A_{1}$ and $A_{2}$ neighborhoods of $x$ and $y$ respectively, has a finite IP-independence 
set of length $n(d)$ such that $\lim _{d \rightarrow \infty} n(d)=\infty$. This means that if $(x, y) \in \mathbf{R} \mathbf{P}^{[\infty]}=$ $\cap_{d \geq 1} \mathbf{R} \mathbf{P}^{[d]}$, then $(x, y) \in \operatorname{Ind}_{f i p}(X, T)$. This is the main reason leading us to define $\infty$-step nilsystems and to study properties of minimal t.d.s. without $\operatorname{Ind}_{f i p}$-pairs.

The third motivation comes from the theory of local complexity in topological dynamics. In [4] the authors introduced the notion of topological complexity for a t.d.s. using open covers, and showed that a t.d.s. is equicontinuous if and only if each nontrivial open cover has a bounded complexity. For a further development, see [21. An interesting, but more difficult question, is to understand t.d.s. with polynomial complexity or extensions of such systems. It appears (this is proved in Section (7) that inverse limits of nilsystems are special systems with polynomial complexity.

1.2. Main results of the paper. In this paper, we study $\infty$-step nilsystems, which are t.d.s. with trivial $\mathbf{R} \mathbf{P}^{[\infty]}$, i.e. $\mathbf{R} \mathbf{P}^{[\infty]}=\cap_{d=1}^{\infty} \mathbf{R} \mathbf{P}^{[d]}=\Delta$, the diagonal. First, we prove that a minimal system is an $\infty$-step nilsystem if and only if it is an inverse limit of minimal nilsystems. Then we study the relation of $\infty$-step nilsystems and independence pairs. It is proved that any minimal system without nontrivial Ind $_{f i p^{-}}$ pairs is an almost one-to-one extension of its maximal $\infty$-step nilfactor. Moreover, a minimal distal system is an $\infty$-step nilsystem if and only if it has no nontrivial Ind $_{\text {fip }}$-pairs. We observe that there are plenty of minimal distal systems which are not $\infty$-step nilsystems, though it is not easy to construct explicit examples.

In addition, we show for any minimal system without nontrivial $\operatorname{Ind}_{\text {fip }}$-pairs that each invariant ergodic measure is measure theoretical isomorphic to the Haar measure on some $\infty$-step nilsystem. We conjecture that such class of systems are uniquely ergodic.

Finally, we prove the topological complexity of an $\infty$-step nilsystem is polynomial for each non-trivial open cover.

1.3. Organization of the paper. We organize the paper as follows. In Section 2 we introduce basic notions and facts we will meet in this article. Then we define $\infty$-step nilsystems in Section 3. In Section 4 we study the relationship between $\infty$ step nilsystems and independence pairs; and in Section 5, we give some examples. In section 6 we study the conjecture concerning unique ergodicity, and state some further ideas and questions. Finally, in Section 7, we discuss the complexity of an $\infty$-step nilsystem. Moreover, we give proofs of some results stated in Section 3 in the Appendix.

Acknowledgements: We thank W. Huang, H.F. Li and B. Kra for useful comments and suggestions.

\section{PRELIMINARIES}

2.1. Topological dynamical systems. A transformation of a compact metric space $\mathrm{X}$ is a homeomorphism of $\mathrm{X}$ to itself. A topological dynamical system (t.d.s.) or 
just a system, is a pair $(X, T)$, where $X$ is a compact metric space and $T: X \rightarrow X$ is a transformation. We use $\rho(\cdot, \cdot)$ to denote the metric in $X$. In the sequel, and if there is no confusion, in any t.d.s. we will always use $T$ to indicate the transformation.

We will also make use of a more general definition of a system. That is, instead of just considering a single transformation $T$, we will consider commuting homeomorphisms $T_{1}, \ldots, T_{k}$ of $X$. We recall some basic definitions and properties of systems in the classical setting of one transformation. Extensions to the general case are straightforward.

A system $(X, T)$ is transitive if there exists $x \in X$ whose orbit $\mathcal{O}(x, T)=\left\{T^{n} x\right.$ : $n \in \mathbb{Z}\}$ is dense in $X$ and such point is called a transitive point. The system is minimal if the orbit of any point is dense in $X$. This property is equivalent to saying that $\mathrm{X}$ and the empty set are the unique closed invariant subsets of $X$.

Let $(X, T)$ be a system and $\mathcal{M}(X)$ be the set of Borel probability measures in $X$. A measure $\mu \in \mathcal{M}(X)$ is $T$-invariant if for any Borel set $B$ of $X, \mu\left(T^{-1} B\right)=$ $\mu(B)$. Denote by $\mathcal{M}(X, T)$ the set of invariant probability measures. A measure $\mu \in \mathcal{M}(X, T)$ is ergodic if for any Borel set $B$ of $X$ satisfying $\mu\left(T^{-1} B \triangle B\right)=0$ we have $\mu(B)=0$ or $\mu(B)=1$. Denote by $\mathcal{M}^{e}(X, T)$ the set of ergodic measures. The system $(X, T)$ is uniquely ergodic if $\mathcal{M}(X, T)$ consists of only one element.

A homomorphism between the t.d.s. $(X, T)$ and $(Y, T)$ is a continuous onto map $\pi: X \rightarrow Y$ which intertwines the actions; one says that $(Y, T)$ is a factor of $(X, T)$ and that $(X, T)$ is an extension of $(Y, T)$. One also refers to $\pi$ as a factor map or an extension and one uses the notation $\pi:(X, T) \rightarrow(Y, T)$. The systems are said to be conjugate if $\pi$ is a bijection. An extension $\pi$ is determined by the corresponding closed invariant equivalence relation $R_{\pi}=\left\{\left(x_{1}, x_{2}\right): \pi\left(x_{1}\right)=\pi\left(x_{2}\right)\right\}=(\pi \times$ $\pi)^{-1} \Delta_{Y} \subset X \times X$, where $\Delta_{Y}$ is the diagonal on $Y$. An extension $\pi:(X, T) \rightarrow(Y, T)$ is almost one-to-one if the $G_{\delta}$ set $X_{0}=\left\{x \in X: \pi^{-1}(\pi(x))=\{x\}\right\}$ is dense.

2.2. Distality and Proximality. Let $(X, T)$ be a t.d.s. A pair $(x, y) \in X \times X$ is a proximal pair if

$$
\inf _{n \in \mathbb{Z}} \rho\left(T^{n} x, T^{n} y\right)=0
$$

and is a distal pair if it is not proximal. Denote by $P(X, T)$ or $P_{X}$ the set of proximal pairs of $(X, T)$. The t.d.s. $(X, T)$ is distal if $(x, y)$ is a distal pair whenever $x, y \in X$ are distinct.

An extension $\pi:(X, T) \rightarrow(Y, S)$ is proximal if $R_{\pi} \subset P(X, T)$ and is distal if $R_{\pi} \cap P(X, T)=\Delta_{X}$. Observe that when $Y$ is trivial (reduced to one point) the map $\pi$ is distal if and only if $(X, T)$ is distal.

2.3. Independence. The notion of independence was firstly introduced and studied in [24, Definition 2.1]. It corresponds to a modification of the notion of interpolating studied in [11, 22] and was considerably discussed in [18, 19]. 
Definition 2.1. Let $(X, T)$ be a t.d.s. Given a tuple $\mathcal{A}=\left(A_{1}, \ldots, A_{k}\right)$ of subsets of $X$ we say that a subset $F \subset \mathbb{Z}_{+}$is an independence set for $\mathcal{A}$ if for any nonempty finite subset $J \subset F$ and any $s=(s(j): j \in J) \in\{1, \ldots, k\}^{J}$ we have

$$
\bigcap_{j \in J} T^{-j} A_{s(j)} \neq \emptyset \text {. }
$$

We shall denote the collection of all independence sets for $\mathcal{A}$ by $\operatorname{Ind}\left(A_{1}, \ldots, A_{k}\right)$ or $\operatorname{Ind} \mathcal{A}$.

A finite subset $F$ of $\mathbb{Z}_{+}$is called a finite IP-set if there exists a finite subset $\left\{p_{1}, p_{2}, \ldots, p_{m}\right\}$ of $\mathbb{N}$ such that

$$
F=F S\left(\left\{p_{i}\right\}_{i=1}^{m}\right)=\left\{p_{i_{1}}+\cdots+p_{i_{k}}: 1 \leq i_{1}<\cdots<i_{k} \leq m\right\} .
$$

Now we define Ind $_{f i p}$-pairs.

Definition 2.2. Let $(X, T)$ be a t.d.s. A pair $\left(x_{1}, x_{2}\right) \in X \times X$ is called an Ind $_{\text {fip }^{-}}$ pair if for any neighborhoods $U_{1}, U_{2}$ of $x_{1}$ and $x_{2}$ respectively, $\operatorname{Ind}\left(U_{1}, U_{2}\right)$ contains arbitrarily long finite IP-sets. Denote by $\operatorname{Ind}_{\text {fip }}(X, T)$ the set of all $\operatorname{Ind}_{\text {fip }}$-pairs of $(X, T)$.

2.4. Parallelepipeds. Let $X$ be a set, $d \geq 1$ be an integer, and write $[d]=$ $\{1,2, \ldots, d\}$. We view $\{0,1\}^{d}$ in one of two ways, either as a sequence $\epsilon=\epsilon_{1} \ldots \epsilon_{d}:=$ $\left(\epsilon_{1}, \ldots, \epsilon_{d}\right)$ of $0^{\prime} \mathrm{s}$ and $1^{\prime} \mathrm{s}$; or as a subset of $[d]$. A subset $\epsilon$ corresponds to the sequence $\left(\epsilon_{1}, \ldots, \epsilon_{d}\right) \in\{0,1\}^{d}$ such that $i \in \epsilon$ if and only if $\epsilon_{i}=1$ for $i \in[d]$. For example, $\mathbf{0}=(0,0, \ldots, 0) \in\{0,1\}^{d}$ is the same as $\emptyset \subset[d]$.

If $\mathbf{n}=\left(n_{1}, \ldots, n_{d}\right) \in \mathbb{Z}^{d}$ and $\epsilon \in\{0,1\}^{d}$, we define

$$
\mathbf{n} \cdot \epsilon=\sum_{i=1}^{d} n_{i} \epsilon_{i} .
$$

If we consider $\epsilon$ as $\epsilon \subset[d]$, then $\mathbf{n} \cdot \epsilon=\sum_{i \in \epsilon} n_{i}$.

We denote $X^{2^{d}}$ by $X^{[d]}$. A point $\mathbf{x} \in X^{[d]}$ can be written in one of two equivalent ways, depending on the context:

$$
\mathbf{x}=\left(x_{\epsilon}: \epsilon \in\{0,1\}^{d}\right)=\left(x_{\epsilon}: \epsilon \subset[d]\right) .
$$

Hence $x_{\emptyset}=x_{\mathbf{0}}$ is the first coordinate of $\mathbf{x}$. As examples, points in $X^{[2]}$ are written

$$
\left(x_{00}, x_{10}, x_{01}, x_{11}\right)=\left(x_{\emptyset}, x_{\{1\}}, x_{\{2\}}, x_{\{1,2\}}\right),
$$

and points in $X^{[3]}$ look like

$$
\begin{aligned}
& \left(x_{000}, x_{100}, x_{010}, x_{110}, x_{001}, x_{101}, x_{011}, x_{111}\right) \\
= & \left(x_{\emptyset}, x_{\{1\}}, x_{\{2\}}, x_{\{1,2\}}, x_{\{3\}}, x_{\{1,3\}}, x_{\{2,3\}}, x_{\{1,2,3\}}\right) .
\end{aligned}
$$

For $x \in X$ we write $x^{[d]}=(x, x, \ldots, x) \in X^{[d]}$. The diagonal of $X^{[d]}$ is $\Delta^{[d]}=$ $\left\{x^{[d]}: x \in X\right\}$. Usually, when $d=1$, we denote the diagonal by $\Delta_{X}$ or $\Delta$ instead of $\Delta^{[1]}$. 
A point $\mathbf{x} \in X^{[d]}$ can be decomposed as $\mathbf{x}=\left(\mathbf{x}^{\prime}, \mathbf{x}^{\prime \prime}\right)$ with $\mathbf{x}^{\prime}, \mathbf{x}^{\prime \prime} \in X^{[d-1]}$, where $\mathbf{x}^{\prime}=\left(x_{\epsilon 0}: \epsilon \in\{0,1\}^{d-1}\right)$ and $\mathbf{x}^{\prime \prime}=\left(x_{\epsilon 1}: \epsilon \in\{0,1\}^{d-1}\right)$. We can also isolate the first coordinate, writing $X_{*}^{[d]}=X^{2^{d}-1}$ and then writing a point $\mathbf{x} \in X^{[d]}$ as $\mathbf{x}=\left(x_{\emptyset}, \mathbf{x}_{*}\right)$, where $\mathbf{x}_{*}=\left(x_{\epsilon}: \epsilon \neq \emptyset\right) \in X_{*}^{[d]}$.

Identifying $\{0,1\}^{d}$ with the set of vertices of the Euclidean unit cube, a Euclidean isometry of the unit cube permutes the vertices of the cube and thus the coordinates of a point $\mathbf{x} \in X^{[d]}$. These permutations are the Euclidean permutations of $X^{[d]}$.

2.5. Dynamical parallelepipeds. We follow definitions from [16].

Let $(X, T)$ be a t.d.s. and $d \geq 1$ be an integer.

We define the set of (dynamical) parallelepipeds of dimension $d, \mathbf{Q}^{[d]}(X)$ (or just $\left.\mathrm{Q}^{[d]}\right)$, as the closure in $X^{[d]}$ of elements of the form

$$
\left(T^{\mathbf{n} \cdot \epsilon} x=T^{n_{1} \epsilon_{1}+\ldots+n_{d} \epsilon_{d}} x: \epsilon=\left(\epsilon_{1}, \ldots, \epsilon_{d}\right) \in\{0,1\}^{d}\right),
$$

where $\mathbf{n}=\left(n_{1}, \ldots, n_{d}\right) \in \mathbb{Z}^{d}$ and $x \in X$. It is important to note that $\mathbf{Q}^{[d]}$ is invariant under the Euclidean permutations of $X^{[d]}$.

As examples, $\mathrm{Q}^{[2]}$ is the closure in $X^{[2]}=X^{4}$ of the set

$$
\left\{\left(x, T^{m} x, T^{n} x, T^{n+m} x\right): x \in X, m, n \in \mathbb{Z}\right\}
$$

and $\mathrm{Q}^{[3]}$ is the closure in $X^{[3]}=X^{8}$ of the set

$$
\left\{\left(x, T^{m} x, T^{n} x, T^{m+n} x, T^{p} x, T^{m+p} x, T^{n+p} x, T^{m+n+p} x\right): x \in X, m, n, p \in \mathbb{Z}\right\} .
$$

Let $\phi:(X, T) \rightarrow(Y, T)$ be a factor map. Define $\phi^{[d]}: X^{[d]} \rightarrow Y^{[d]}$ by $\left(\phi^{[d]} \mathbf{x}\right)_{\epsilon}=\phi x_{\epsilon}$ for every $\mathbf{x} \in X^{[d]}$ and every $\epsilon \subset[d]$. The diagonal transformation of $X^{[d]}$ is the map $T^{[d]}$. We define face transformations inductively as follows: Let $T^{[0]}=T$, $T_{1}^{[1]}=\mathrm{id} \times T$. If $\left\{T_{j}^{[d-1]}\right\}_{j=1}^{d-1}$ is already defined, then set

$$
\begin{gathered}
T_{j}^{[d]}=T_{j}^{[d-1]} \times T_{j}^{[d-1]}, j \in\{1,2, \ldots, d-1\}, \\
T_{d}^{[d]}=\mathrm{id}^{[d-1]} \times T^{[d-1]} .
\end{gathered}
$$

It is easy to see that for $j \in[d]$, the face transformation $T_{j}^{[d]}: X^{[d]} \rightarrow X^{[d]}$ can be defined, for every $\mathbf{x} \in X^{[d]}$ and $\epsilon \subset[d]$, by

$$
T_{j}^{[d]} \mathbf{x}= \begin{cases}\left(T_{j}^{[d]} \mathbf{x}\right)_{\epsilon}=T x_{\epsilon}, & j \in \epsilon ; \\ \left(T_{j}^{[d]} \mathbf{x}\right)_{\epsilon}=x_{\epsilon}, & j \notin \epsilon .\end{cases}
$$

The face group of dimension $d$ is the group $\mathcal{F}^{[d]}(X)$ of transformations of $X^{[d]}$ spanned by the face transformations. The parallelepiped group of dimension $d$ is the group $\mathcal{G}^{[d]}(X)$ spanned by the diagonal transformation and the face transformations. We often write $\mathcal{F}^{[d]}$ and $\mathcal{G}^{[d]}$ instead of $\mathcal{F}^{[d]}(X)$ and $\mathcal{G}^{[d]}(X)$, respectively. For $\mathcal{G}^{[d]}$ and $\mathcal{F}^{[d]}$, we use similar notations to that used for $X^{[d]}$ : Namely, an element of either of these groups is written as $S=\left(S_{\epsilon}: \epsilon \in\{0,1\}^{d}\right)$. In particular, $\mathcal{F}^{[d]}=\left\{S \in \mathcal{G}^{[d]}\right.$ : $\left.S_{\emptyset}=\mathrm{id}\right\}$. 
For convenience, we denote the orbit closure of $\mathbf{x} \in X^{[d]}$ under $\mathcal{F}^{[d]}$ by $\overline{\mathcal{F}}^{[d]}(\mathbf{x})$, instead of $\overline{\mathcal{O}\left(\mathbf{x}, \mathcal{F}^{[d]}\right)}$.

It is easy to verify that $\mathbf{Q}^{[d]}$ is the closure in $X^{[d]}$ of

$$
\left\{S x^{[d]}: S \in \mathcal{F}^{[d]}, x \in X\right\} .
$$

If $(X, T)$ is a transitive system and $x$ a transitive point, then $\mathbf{Q}^{[d]}$ is the closed orbit of $x^{[d]}$ under the group $\mathcal{G}^{[d]}$. Moreover, it is easy to get that

$$
\mathbf{Q}^{[d]}=\overline{\left\{\left(T^{\mathbf{n} \cdot \epsilon} x\right)_{\epsilon \in\{0,1\}^{d}}: \mathbf{n} \in \mathbb{N}^{d}, x \in X\right\}} .
$$

2.6. Nilmanifolds and nilsystems. Let $G$ be a group. For $g, h \in G$ and $A, B \subset$ $G$, we write $[g, h]=g h g^{-1} h^{-1}$ for the commutator of $g$ and $h$ and $[A, B]$ for the subgroup spanned by $\{[a, b]: a \in A, b \in B\}$. The commutator subgroups $G_{j}, j \geq 1$, are defined inductively by setting $G_{1}=G$ and $G_{j+1}=\left[G_{j}, G\right]$. Let $d \geq 1$ be an integer. We say that $G$ is $d$-step nilpotent if $G_{d+1}$ is the trivial subgroup.

Let $G$ be a $d$-step nilpotent Lie group and $\Gamma$ be a discrete cocompact subgroup of $G$. The compact manifold $X=G / \Gamma$ is called a $d$-step nilmanifold. The group $G$ acts on $X$ by left translations and we write this action as $(g, x) \mapsto g x$. The Haar measure $\mu$ of $X$ is the unique probability measure on $X$ invariant under this action. Let $\tau \in G$ and $T$ be the transformation $x \mapsto \tau x$ of $X$. Then $(X, \mu, T)$ is called a $d$-step nilsystem. In the topological setting we omit the measure and just say that $(X, T)$ is a $d$-step nilsystem.

We will need to use inverse limits of nilsystems, so we recall the definition of a sequential inverse limit of systems. If $\left(X_{i}, T_{i}\right)_{i \in \mathbb{N}}$ are systems with $\operatorname{diam}\left(X_{i}\right) \leq 1$ and $\pi_{i}: X_{i+1} \rightarrow X_{i}$ are factor maps, the inverse limit of the systems is defined to be the compact subset of $\prod_{i \in \mathbb{N}} X_{i}$ given by $\left\{\left(x_{i}\right)_{i \in \mathbb{N}}: \pi_{i}\left(x_{i+1}\right)=x_{i}\right\}$, and we denote it by $\lim \left(X_{i}, T_{i}\right)_{i \in \mathbb{N}}$. It is a compact metric space endowed with the distance $\rho\left(\left(x_{i}\right)_{i \in \mathbb{N}},\left(y_{i}\right)_{i \in \mathbb{N}}^{\longleftarrow}\right)=\sum_{i \in \mathbb{N}} 1 / 2^{i} \rho_{i}\left(x_{i}, y_{i}\right)$, where $\rho_{i}$ is the metric in $X_{i}$. We note that the maps $T_{i}$ induce naturally a transformation $T$ on the inverse limit.

The following structure theorem characterizes inverse limits of nilsystems using dynamical parallelepipeds.

Theorem 2.3 (Host-Kra-Maass). [16, Theorem 1.2.] Assume that $(X, T)$ is a transitive topological dynamical system and let $d \geq 2$ be an integer. The following properties are equivalent:

(1) If $\mathbf{x}, \mathbf{y} \in \mathbf{Q}^{[d]}$ have $2^{d}-1$ coordinates in common, then $\mathbf{x}=\mathbf{y}$.

(2) If $x, y \in X$ are such that $(x, y, \ldots, y) \in \mathbf{Q}^{[d]}$, then $x=y$.

(3) $X$ is an inverse limit of $(d-1)$-step minimal nilsystems.

A transitive system satisfying one of the equivalent properties above is called a system of order $(d-1)$. 


\section{3. $\infty$-STEP NILSYSTEMS}

3.1. Regionally proximal relation of order $d$. First we recall a fundamental relation introduced in [16] allowing to characterize maximal nilfactors in [16] (for minimal distal systems) and in [29] (for general minimal systems).

Definition 3.1. Let $(X, T)$ be a system and let $d \in \mathbb{N}$. The points $x, y \in X$ are said to be regionally proximal of order $d$ if for any $\delta>0$, there exist $x^{\prime}, y^{\prime} \in X$ and a vector $\mathbf{n}=\left(n_{1}, \ldots, n_{d}\right) \in \mathbb{Z}^{d}$ such that $\rho\left(x, x^{\prime}\right)<\delta, \rho\left(y, y^{\prime}\right)<\delta$, and

$$
\rho\left(T^{\mathbf{n} \cdot \epsilon} x^{\prime}, T^{\mathbf{n} \cdot \epsilon} y^{\prime}\right)<\delta \text { for any nonempty } \epsilon \subset[d] .
$$

In other words, there exists $S \in \mathcal{F}^{[d]}$ such that $\rho\left(S_{\epsilon} x^{\prime}, S_{\epsilon} y^{\prime}\right)<\delta$ for every $\epsilon \neq \emptyset$. The set of regionally proximal pairs of order $d$ is denoted by $\mathbf{R P}^{[d]}$ (or by $\mathbf{R P}^{[d]}(X, T$ ) in case of ambiguity), and is called the regionally proximal relation of order $d$.

It is easy to see that $\mathbf{R} \mathbf{P}^{[d]}$ is a closed and invariant relation. Observe that

$$
\mathbf{P}(X, T) \subseteq \ldots \subseteq \mathbf{R} \mathbf{P}^{[d+1]} \subseteq \mathbf{R} \mathbf{P}^{[d]} \subseteq \ldots \mathbf{R P}^{[2]} \subseteq \mathbf{R} \mathbf{P}^{[1]}=\mathbf{R} \mathbf{P}(X, T)
$$

The following theorems proved in [16] (for minimal distal systems) and in [29] (for general minimal systems) tell us conditions under which $(x, y)$ belongs to $\mathbf{R P}^{[d]}$ and the relation between $\mathbf{R} \mathbf{P}^{[d]}$ and $d$-step nilsystems.

Theorem 3.2. Let $(X, T)$ be a minimal system and let $d \in \mathbb{N}$. Then

(1) $(x, y) \in \mathbf{R P}^{[d]}$ if and only if $(x, y, \ldots, y) \in \mathbf{Q}^{[d+1]}$ if and only if $(x, y, \ldots, y) \in$ $\overline{\mathcal{F}^{[d+1]}}\left(x^{[d+1]}\right)$.

(2) $\mathbf{R} \mathbf{P}^{[d]}$ is an equivalence relation.

(3) $(X, T)$ is a system of order $d$ if and only if $\mathbf{R} \mathbf{P}^{[d]}=\Delta_{X}$.

3.2. $\infty$-step Nilsystems. The regionally proximal relation of order $d$ allows to construct the maximal $d$-step nilfactor of a system. That is, any factor of order $d$ (inverse limit of $d$-step minimal nilsystems) factorize through this system.

Theorem 3.3. [29] Let $\pi:(X, T) \rightarrow(Y, T)$ be a factor map between minimal systems and let $d \in \mathbb{N}$. Then,

(1) $\pi \times \pi\left(\mathbf{R P}^{[d]}(X, T)\right)=\mathbf{R P}^{[d]}(Y, T)$.

(2) $(Y, T)$ is a system of order $d$ if and only if $\mathbf{R P}^{[d]}(X, T) \subset R_{\pi}$.

In particular, the quotient of $(X, T)$ under $\mathbf{R P}^{[d]}(X, T)$ is the maximal d-step nilfactor of $X$ (i.e. the maximal factor of order $d$ ).

It follows that for any minimal system $(X, T)$,

$$
\mathbf{R P}^{[\infty]}=\bigcap_{d \geq 1} \mathbf{R P}^{[d]}
$$


is a closed invariant equivalence relation (we write $\mathbf{R P}^{[\infty]}(X, T)$ in case of ambiguity). Now we formulate the definition of $\infty$-step nilsystems or systems of order $\infty$.

Definition 3.4. A minimal system $(X, T)$ is an $\infty$-step nilsystem or a system of $\operatorname{order} \infty$, if the equivalence relation $\mathbf{R P}^{[\infty]}$ is trivial, i.e. coincides with the diagonal.

Remark 3.5. Similar to Theorem 3.3 , one can show that the quotient of a minimal system $(X, T)$ under $\mathbf{R} \mathbf{P}^{[\infty]}$ is the maximal $\infty$-step nilfactor of $(X, T)$.

Let $(X, T)$ be a minimal system. It is easy to see that if $(X, T)$ is an inverse limit of minimal nilsystems, then $(X, T)$ is an $\infty$-step nilsystem. Conversely, if $(X, T)$ is a minimal $\infty$-step nilsystem, then $\mathbf{R P}^{[\infty]}=\Delta_{X}$. For any integer $d \geq 1$ let $\left(X_{d}, T\right)$ be the quotient of $(X, T)$ under $\mathbf{R P}^{[d]}$. Then $(X, T)=\lim \left(X_{d}, T\right)_{d \in \mathbb{N}}$ as $\Delta_{X}=\mathbf{R} \mathbf{P}^{[\infty]}=\bigcap_{d \geq 1} \mathbf{R P}^{[d]}$. In fact we can show more as the following theorem says.

Theorem 3.6. A minimal system is an $\infty$-step nilsystem if and only if it is an inverse limit of minimal nilsystems.

Proof. It remains to prove that if $(X, T)$ is a minimal $\infty$-step nilsystem, then it is an inverse limit of minimal nilsystems. First we may assume that $(X, T)=$ $\lim _{\longleftarrow}\left(X_{d}, T\right)_{d \in \mathbb{N}}$, where $X_{d}=X / \mathbf{R P}^{[d]}$ for any $d \geq 1$. By Theorem 2.3 , for any $d \geq 1$, $\left.\overleftarrow{(X}_{d}, T\right)$ is an inverse limit of minimal $d$-step nilsystems.

We need the following claim.

Claim: Let $(Y, S)$ be a minimal system, and let $\left(Y_{i}, S\right)$ be factors of $(Y, S)$ which are $k_{i}$-step nilsystems, where $1 \leq i \leq n$ and $\max \left\{k_{i}: 1 \leq i \leq n\right\}=k$. Then there exists a $k$-step nilsystem $(Z, S)$ such that it is a factor of $(Y, S)$ and is an extension of $\left(Y_{i}, S\right)$ for $1 \leq i \leq n$.

Proof of Claim: Let $\pi_{i}$ be the factor map between $(Y, S)$ and $\left(Y_{i}, S\right)$ and assume $\left(Y_{i}, S\right)$ has the form of $\left(H_{i} / \Gamma_{i}, h_{i}\right)$, where $h_{i} \in H_{i}$ and $S$ is the left translation by $h_{i}$ on $Y_{i}$. Set $G=H_{1} \times \cdots \times H_{n}, \Gamma=\Gamma_{1} \times \cdots \Gamma_{n}$ and $g=\left(h_{1}, \ldots, h_{n}\right)$. Then $G$ is a $k$-step nilpotent Lie group and $\Gamma$ is a discrete uniform subgroup of $G$. Let $S: G / \Gamma \rightarrow G / \Gamma$ be the transformation $x \mapsto g x$. Choose any point $y \in Y$ and let $Z=\overline{\left\{g^{n}\left(\pi_{1}(y), \ldots, \pi_{n}(y)\right): n \in \mathbb{Z}\right\}} \subset G / \Gamma$ be the orbit closure of $\left(\pi_{1}(y), \ldots, \pi_{n}(y)\right)$ under $S$. Since nilsystems are distal, $(Z, S)$ is minimal. Moreover it is a $k$-step nilsystem [25]. And of course, $(Z, S)$ is a factor of $(Y, S)$ and it is an extension of $\left(Y_{i}, S\right)$ for $1 \leq i \leq n$.

Now we show that $(X, T)$ is an inverse limit of minimal nilsystems using previous claim. As $\left(X_{1}, T\right)$ is a system of order 1 , it is an inverse limit of some 1-step nilsystems $\left(\left(X_{1}\right)_{i}, T\right)_{i \in \mathbb{N}}$ by Theorem 2.3. Similarly, as $\left(X_{2}, T\right)$ is a system of order 2 , it is an inverse limit of some 2 -step nilsystems $\left(\left(X_{2}\right)_{i}, T\right)_{i \in \mathbb{N}}$. Note that all $\left(\left(X_{1}\right)_{i}, T\right)_{i \in \mathbb{N}}$ 
and $\left(\left(X_{2}\right)_{i}, T\right)_{i \in \mathbb{N}}$ are factors of $X_{2}$. By the above claim, we may reconstruct 2step nilsystems $\left(\left(X_{2}\right)_{i}, T\right)_{i \in \mathbb{N}}$ such that for all $i \in \mathbb{N},\left(\left(X_{2}\right)_{i+1}, T\right)$ is an extension of $\left(\left(X_{1}\right)_{1}, T\right),\left(\left(X_{1}\right)_{2}, T\right), \ldots,\left(\left(X_{1}\right)_{i+1}, T\right)$ and $\left(\left(X_{2}\right)_{1}, T\right), \ldots,\left(\left(X_{2}\right)_{i}, T\right)$.

Similarly and inductively, for any given $k \in \mathbb{N}\left(X_{k}, T\right)$ can be written as the inverse limit of some $k$-step nilsystems $\left(\left(X_{k}\right)_{i}, T\right)_{i \in \mathbb{N}}$ satisfying that for all $i \in \mathbb{N}$, $\left(\left(X_{k+1}\right)_{i}, T\right)$ is an extension of $\left(\left(X_{k}\right)_{i}, T\right)$.

Since $(X, T)$ is the inverse limit of $\left(X_{k}, T\right)_{k \in \mathbb{N}}, \bigcup_{k \in \mathbb{N}} C\left(X_{k}\right)$ is dense in $C(X)$. And as $\left(X_{k}, T\right)$ is the inverse limit of $\left(\left(X_{k}\right)_{i}, T\right)_{i \in \mathbb{N}}$, we have that $\bigcup_{k \in \mathbb{N}} \bigcup_{i \in \mathbb{N}} C\left(\left(X_{k}\right)_{i}\right)$ is dense in $C(X)$. So we may choose a sequence of $\left(k_{n}, i_{n}\right)_{n \in \mathbb{N}} \subset \mathbb{N} \times \mathbb{N}$ with $k_{n}<k_{n+1}, i_{n}<i_{n+1}$ such that $\bigcup_{n \in \mathbb{N}} C\left(\left(X_{k_{n}}\right)_{i_{n}}\right)$ is dense in $C(X)$. Thus $(X, T)$ is the inverse limit of $\left(\left(X_{k_{n}}\right)_{i_{n}}, T\right)_{n \in \mathbb{N}}$. That is, $(X, T)$ is an inverse limit of minimal nilsystems. This completes the proof of the theorem.

Since minimal nilsystems are uniquely ergodic, it is easy to see that minimal $\infty$-step nilsystems are also uniquely ergodic.

3.3. About $\mathbf{R} \mathbf{P}^{[\infty]}=\mathbf{R} \mathbf{P}^{[d]}$. Observe that if $\mathbf{Q}^{[d+1]}=\mathbf{Q}^{[d]} \times \mathbf{Q}^{[d]}$ then $\mathbf{R} \mathbf{P}^{[d-1]}=$ $\mathbf{R P}^{[d]}$. Indeed, if $(x, y, \ldots, y)$ and $(y, \ldots, y) \in \mathbf{Q}^{[d]}$, then $(x, y, \ldots, y, y, \ldots, y) \in$ $\mathbf{Q}^{[d+1]}$. Moreover, the system is weakly-mixing and thus $\mathbf{R} \mathbf{P}^{[d]}=X \times X$ for any $d \geq 1$, as shows the following proposition.

Proposition 3.7. Let $(X, T)$ be a minimal system. If $\mathbf{Q}^{[d+1]}=\mathbf{Q}^{[d]} \times \mathbf{Q}^{[d]}$ for some $d \in \mathbb{N}$ then $X$ is weakly-mixing and hence $\mathbf{Q}^{[d]}=X^{[d]}$ for any $d \in \mathbb{N}$.

Proof. Let $x, y, a \in X$. By minimality $(x, y, x, y, \ldots, x, y) \in \mathbf{Q}^{[d]}$ and by hypothesis the point $\mathbf{x}=(x, y, x, y, \ldots, x, y, a, \ldots, a) \in \mathbf{Q}^{[d+1]}$. If $d=1,(x, y, a, a) \in \mathbf{Q}^{[2]}$ and then $(x, y) \in \mathbf{R} \mathbf{P}^{[1]}$. For any integer $d>1$, applying Euclidean permutations, we get that $\mathbf{y}=(x, y, \ldots, x, y, a, \ldots, a, x, y \ldots, x, y, a, \ldots, a) \in \mathbf{Q}^{[d+1]}$ too. Considering the first half of $\mathbf{y}$ and iterating the process we finish in the case $d=1$. We conclude $\mathbf{R} \mathbf{P}^{[1]}=X \times X$ and the result follows.

Now, if $\mathbf{R} \mathbf{P}^{[d-1]}=\mathbf{R} \mathbf{P}^{[d]}$ for some $d$ the following theorem states that all regionally proximal relations of higher order coincide and thus $\mathbf{R} \mathbf{P}^{[\infty]}=\mathbf{R} \mathbf{P}^{[d-1]}$. This result is natural but its proof is somewhat involved, so we leave it for the appendix. For the definition of $Z_{d}$ see Section 7 .

Theorem 3.8. (1) Let $(X, T)$ be a minimal system. If $\mathbf{R} \mathbf{P}^{[d]}=\mathbf{R} \mathbf{P}^{[d+1]}$ for some $d \in \mathbb{N}$, then $\mathbf{R P}^{[n]}=\mathbf{R} \mathbf{P}^{[d]}$ for all $n \geq d$.

(2) Let $(X, \mathcal{B}, \mu, T)$ be an ergodic measure preserving system. If $Z_{d}=Z_{d+1}$ for some $d \in \mathbb{N}$, then $Z_{n}=Z_{d}$ for each $n \geq d$.

(3) Let $(X, T)$ be a minimal system and $\mu$ be an ergodic Borel probability measure on $X$. If $Z_{n}$ is isomorphic (with respect to the corresponding invariant measure) to $X_{n}=X / \mathbf{R P}^{[n]}$ for some $n \in \mathbb{N}$, then $Z_{k}$ is isomorphic to $X_{k}=X / \mathbf{R P}^{[k]}$ for all $k \leq n$. 
4. The structure of minimal Systems without nOntrivial Ind $_{\text {fip } \text {-Pairs }}$

In this section we discuss the structure of minimal systems without nontrivial Ind $_{\text {fip }}$-pairs. We will show that such systems are almost one-to-one extensions of their maximal $\infty$-step nilfactors.

4.1. A criterion to be an $\operatorname{Ind}_{f i p}$-pair. First we characterize $\operatorname{Ind}_{f i p}$-pairs using dynamical parallelepipeds.

Let $(X, T)$ be a transitive system. It is easy to check that $\mathbf{x}=\left(x_{\epsilon}: \epsilon \subset[d]\right) \in \mathbf{Q}^{[d]}$ if and only if for any neighborhood $U_{\epsilon}$ of $x_{\epsilon}$ respectively, there exist positive integers $\mathbf{n}=\left(n_{1}, n_{2}, \ldots, n_{d}\right) \in \mathbb{N}^{d}$ such that $\bigcap_{\epsilon \subset[d]} T^{-\mathbf{n} \cdot \epsilon} U_{\epsilon} \neq \emptyset$. Moreover, the point in $\bigcap_{\epsilon \subset[d]} T^{-\mathbf{n} \cdot \epsilon} U_{\epsilon}$ can be chosen to be in the orbit of a transitive point.

Lemma 4.1. Let $(X, T)$ be a transitive t.d.s. and $\left(x_{1}, x_{2}\right) \in X \times X$ with $x_{1} \neq x_{2}$. Then, $\left\{x_{1}, x_{2}\right\}^{[d]} \subset \mathbf{Q}^{[d]}$ for all integer $d \geq 1$ if and only if $\left(x_{1}, x_{2}\right)$ is an $\operatorname{Ind}_{\text {fip-pair. }}$

Proof. Let $\left(x_{1}, x_{2}\right) \in X \times X$ with $x_{1} \neq x_{2}$. First, we assume $\left\{x_{1}, x_{2}\right\}^{[d]} \subset \mathbf{Q}^{[d]}$ for all integer $d \geq 1$.

Let $U_{1}, U_{2}$ be neighborhoods of $x_{1}$ and $x_{2}$ respectively and fix $d \in \mathbb{N}$. We show there exist positive integers $\left\{p_{1}, p_{2}, \ldots, p_{d}\right\}$ such that

$$
F=F S\left(\left\{p_{i}\right\}_{i=1}^{d}\right)=\left\{p_{i_{1}}+\cdots+p_{i_{k}}: 1 \leq i_{1}<\cdots<i_{k} \leq d\right\}
$$

belongs to $\operatorname{Ind}\left(U_{1}, U_{2}\right)$.

Since $\left\{x_{1}, x_{2}\right\}^{[d]}$ has $2^{2^{d}}$ elements we write it $\left\{\mathbf{x}_{\eta}: \eta \subset\left[2^{d}\right]\right\}$. Now, for any $\epsilon \subset[d]$ and $\eta \subset\left[2^{d}\right]$, let $x_{\epsilon \eta}=\left(\mathbf{x}_{\eta}\right)_{\epsilon}$ and construct the point $\mathbf{x}=\left(x_{\epsilon \eta}: \epsilon \subset[d], \eta \subset\left[2^{d}\right]\right) \in$ $\left\{x_{1}, x_{2}\right\}^{\left[2^{d}+d\right]}$. Clearly, if we choose an identification of coordinates, we can write $\mathbf{x}=\left(x_{\rho}: \rho \subset\left[2^{d}+d\right]\right)$. For any $\rho \subset\left[2^{d}+d\right]$, let $U_{\rho}=U_{i}$ if $x_{\rho}=x_{i}, i=1,2$. Then the product set $\bigotimes_{\rho \subset\left[2^{d}+d\right]} U_{\rho}$ is a neighborhood of $\mathbf{x}$. Since, by hypothesis, $\mathbf{x} \in \mathbf{Q}^{\left[2^{d}+d\right]}$, then there exist $x \in X, p_{0} \in \mathbb{N}$ and $\mathbf{p}=\left\{p_{1}, \ldots, p_{2^{d}+d}\right\} \subset \mathbb{N}$ such that for any $\rho \subset\left[2^{d}+d\right], T^{p_{0}+\mathbf{p} \cdot \rho} x \in U_{\rho}$.

We show that the finite IP set $F$ generated by $\left\{p_{1}, p_{2}, \ldots, p_{d}\right\}$ belongs to $\operatorname{Ind}\left(U_{1}, U_{2}\right)$. For any $s \in\{1,2\}^{2^{d}}=\{1,2\}^{[d]}$, since $\left\{x_{s(\epsilon)}: \epsilon \subset[d]\right\} \in\left\{x_{1}, x_{2}\right\}^{[d]}$, there exists $\eta \subset\left[2^{d}\right]$ such that $\mathbf{x}_{\eta}=\left\{x_{s(\epsilon)}: \epsilon \subset[d]\right\}$, i.e. for any $\epsilon \subset[d], x_{\epsilon \eta}=x_{s(\epsilon)}$. Let $y=T^{p_{0}+\sum_{i \in \eta} p_{i+d}} x$. Then, for any $\epsilon \subset[d], T^{\sum_{i \in \epsilon} p_{i}} y=T^{p_{0}+\mathbf{p} \cdot(\epsilon \eta)} x \in U_{\epsilon \eta}=U_{s(\epsilon)}$. So

$$
\bigcap_{\epsilon \subset[d]} T^{-\sum_{i \in \epsilon} p_{i}} U_{s(\epsilon)} \neq \emptyset,
$$

and $F$ belongs to $\operatorname{Ind}\left(U_{1}, U_{2}\right)$.

Now assume that $\left(x_{1}, x_{2}\right)$ is an $\operatorname{Ind}_{f i p}$-pair. That is, for any neighborhood $U_{1} \times U_{2}$ of $\left(x_{1}, x_{2}\right)$, any $d \in \mathbb{N}$ and any $s \in\{1,2\}^{[d]}$, there are positive integers $p_{1}, \ldots, p_{d}$ such that $\bigcap_{\epsilon \subset[d]} T^{-\sum_{i \in \epsilon} p_{i}} U_{s(\epsilon)} \neq \emptyset$. Let $x \in \bigcap_{\epsilon \subset[d]} T^{-\sum_{i \in \epsilon} p_{i}} U_{s(\epsilon)}$. Then $T^{\sum_{i \in \epsilon} p_{i}} x \in U_{s(\epsilon)}$ for any $\epsilon \subset[d]$. This implies that $\left\{x_{1}, x_{2}\right\}^{[d]} \subset \mathbf{Q}^{[d]}$. 
The following lemma is a useful application of the previous lemma.

Lemma 4.2. Let $(X, T)$ be a transitive system, $x_{1} \in X$ be a transitive point and $d \geq 1$ be an integer. Suppose that $\left(x_{2}, x_{1}, \ldots, x_{1}\right) \in \mathbf{Q}^{[d]}$ for some $x_{2} \in X$ and that $\pi_{1}: A \rightarrow X$ is semi-open, where $A=\overline{\operatorname{orb}\left(\left(x_{1}, x_{2}\right), T \times T\right)}$ and $\pi_{1}$ is the projection to the first coordinate. Then $\left\{x_{1}, x_{2}\right\}^{[d]} \subset \mathbf{Q}^{[d]}$.

Proof. If $X$ is finite, then the lemma holds. Thus we assume that $X$ is infinite. We first prove the following claim.

Claim: If $\mathbf{x}=\left(x_{1}, \mathbf{a}_{*}\right) \in\left\{x_{1}, x_{2}\right\}^{[d]} \cap \mathbf{Q}^{[d]}$, then $\left(x_{2}, \mathbf{a}_{*}\right) \in \mathbf{Q}^{[d]}$.

Let $U_{1}$ and $U_{2}$ be neighborhoods of $x_{1}$ and $x_{2}$ respectively. Since $\pi_{1}$ is semi-open and $X$ is infinite, then $V_{1}=\operatorname{int}\left(\pi_{1}\left(U_{1} \times U_{2} \cap A\right)\right) \neq \emptyset$ and infinite. Set $V_{2}=U_{2}$.

Let $s=(s(\epsilon): \epsilon \subset[d]) \in\{1,2\}^{[d]}$ such that $\mathbf{x}=\left(x_{s(\epsilon)}: \epsilon \subset[d]\right)$. From the hypothesis, $\mathbf{x}=\left(x_{1}, \mathbf{a}_{*}\right) \in\left\{x_{1}, x_{2}\right\}^{[d]} \cap \mathbf{Q}^{[d]}$, then there exist positive integers $n_{0}, n_{1}, \ldots, n_{d}$ such that $T^{n_{0}+\mathbf{n} \cdot \epsilon} x_{1} \in V_{s(\epsilon)}$ for each $\epsilon \subset[d]$, i.e. $\bigcap_{\epsilon \subset[d]} T^{-\mathbf{n} \cdot \epsilon} V_{s(\epsilon)} \neq \emptyset$, where $\mathbf{n}=\left(n_{1}, \ldots, n_{d}\right)$.

Let $W_{1}=\bigcap_{\epsilon \subset[d]} T^{-\mathbf{n} \cdot \epsilon} V_{s(\epsilon)} \subset V_{1}$ and $W_{2}=V_{2}=U_{2}$. Since $W_{1} \times W_{2} \cap A \neq \emptyset$, there exists a positive integer $M$ such that $(T \times T)^{M}\left(x_{1}, x_{2}\right) \in W_{1} \times W_{2}$. And, since $\left(x_{2}, x_{1}, \ldots, x_{1}\right) \in \mathrm{Q}^{[d]}$, there exist positive integers $m_{0}, m_{1}, \ldots, m_{d}$ such that $T^{m_{0}} x_{1} \in T^{-M} W_{2}=T^{-M} U_{2}$, and $T^{m_{0}+\mathbf{m} \cdot \eta} x_{1} \in T^{-M} W_{1}$ for all $\eta \subset[d] \backslash\{\emptyset\}$, where $\mathbf{m}=\left(m_{1}, \ldots, m_{d}\right)$. It follows that $T^{-m_{0}-M} U_{2} \cap \bigcap_{\eta \subset[d] \backslash\{\emptyset\}} T^{-m_{0}-M-\mathbf{m} \cdot \eta} W_{1} \neq \emptyset$. That is,

Thus,

$$
U_{2} \cap \bigcap_{\eta \subset[d] \backslash\{\emptyset\}} T^{-\mathbf{m} \cdot \eta} \bigcap_{\epsilon \subset[d]} T^{-\mathbf{n} \cdot \epsilon} V_{s(\epsilon)} \neq \emptyset .
$$

$$
U_{2} \cap \bigcap_{\eta \subset[d] \backslash\{\emptyset\}} T^{-\mathbf{m} \cdot \eta} \bigcap_{\epsilon \subset[d]} T^{-\mathbf{n} \cdot \epsilon} U_{s(\epsilon)} \neq \emptyset .
$$

Set $p_{i}=n_{i}+m_{i}$ for $1 \leq i \leq d$, and $\mathbf{p}=\left(p_{1}, \ldots, p_{d}\right)$. Then we have

$$
U_{2} \cap \bigcap_{\eta \subset[d] \backslash\{\emptyset\}} T^{-\mathbf{p} \cdot \eta} U_{s(\eta)} \neq \emptyset,
$$

and so we get that $\left(x_{2}, \mathbf{a}_{*}\right) \in \mathbf{Q}^{[d]}$. The proof of the claim is completed.

For any $\mathbf{x} \in\left\{x_{1}, x_{2}\right\}^{[d]}$, let $l(\mathbf{x})$ be the number of $x_{2}$ 's appearing in $\mathbf{x}$. We prove this lemma by induction on $l(\mathbf{x})$.

If $l(\mathbf{x})=0$, then obviously $\mathbf{x}=x_{1}^{[d]} \in \mathbf{Q}^{[d]}$. Suppose the lemma holds when $l(\mathbf{x}) \leq k$, i.e. $\mathbf{x} \in \mathbf{Q}^{[d]}$ if $l(\mathbf{x}) \leq k$. Now, for $l(\mathbf{x})=k+1$ without loss of generality we write $\mathbf{x}=\left(x_{2}, \mathbf{a}_{*}\right)$. Since $l\left(\left(x_{1}, \mathbf{a}_{*}\right)\right)=k$, we have by hypothesis that $\left(x_{1}, \mathbf{a}_{*}\right) \in \mathbf{Q}^{[d]}$. Thus from the claim we get that $\mathbf{x}=\left(x_{2}, \mathbf{a}_{*}\right) \in \mathbf{Q}^{[d]}$. The proof of this lemma is completed. 
The following corollary extends Corollaries 4.2. and 4.3. from [16], and the comment right after, that were only proved in the distal case.

Corollary 4.3. Let $(X, T)$ be a minimal t.d.s., $x_{1}, x_{2} \in X$ and $d \geq 1$ an integer. If $\left(x_{1}, x_{2}\right) \in \mathbf{R P}^{[d]}$ and $\left(x_{1}, x_{2}\right)$ is a $T \times T$-minimal point, then $\left\{x_{1}, x_{2}\right\}^{[d+1]} \subset \mathbf{Q}^{[d+1]}$.

Proof. Let $A=\overline{\operatorname{orb}\left(\left(x_{1}, x_{2}\right), T \times T\right)}$. Since $(A, T \times T)$ and $(X, T)$ are minimal, the projection $\pi_{1}: A \rightarrow X$ is semi-open. By Theorem $3.2,\left(x_{2}, x_{1}, \ldots, x_{1}\right) \in \mathbf{Q}^{[d+1]}$, so $\left\{x_{1}, x_{2}\right\}^{[d+1]} \subset \mathbf{Q}^{[d+1]}$ by Lemma 4.2.

By the above discussion, we get the following criterion to be a $\operatorname{Ind}_{f i p}$-pair.

Corollary 4.4. Let $(X, T)$ be a minimal system and $\left(x_{1}, x_{2}\right) \in \mathbf{R P}^{[\infty]} \backslash \Delta_{X}=$ $\bigcap_{d \geq 1} \mathbf{R P}^{[d]} \backslash \Delta_{X}$. If $\left(x_{1}, x_{2}\right)$ is $T \times T$-minimal or the projection

$$
\pi_{1}: \overline{\operatorname{orb}\left(\left(x_{1}, x_{2}\right), T \times T\right)} \rightarrow X
$$

is semi-open, then $\left(x_{1}, x_{2}\right)$ is an $\operatorname{Ind}_{\text {fip-pair. }}$

Proof. If $\left(x_{1}, x_{2}\right)$ is $T \times T$-minimal, by Corollary 4.3 , we have $\left\{x_{1}, x_{2}\right\}^{[d]} \subset \mathbf{Q}^{[d]}$ for every integer $d \geq 1$. Now, if the projection $\pi_{1}: \overline{\operatorname{orb}\left(\left(x_{1}, x_{2}\right), T \times T\right)} \rightarrow X$ is semiopen, then, since $\left(x_{1}, x_{2}\right) \in \mathbf{R} \mathbf{P}^{[\infty]}$, we have $\left(x_{2}, x_{1}, \ldots, x_{1}\right) \in \mathbf{Q}^{[d]}$ for every $d \geq 1$. Hence, by Lemma 4.2, we also have $\left\{x_{1}, x_{2}\right\}^{[d]} \subset \mathbf{Q}^{[d]}$ for every integer $d \geq 1$.

By Lemma 4.1, the proof is completed.

4.2. The structure of minimal systems without nontrivial $\operatorname{Ind}_{f i p}$-pairs. The following is the main result of this section.

Theorem 4.5. Let $(X, T)$ be a minimal system. If $X$ does not contain any non-

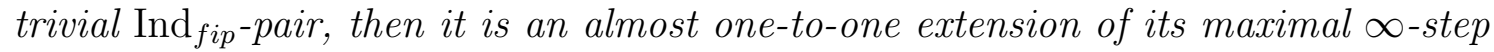
nilfactor.

To prove this theorem we need some preparation. Every extension of minimal systems can be lifted to an open extension by almost one-to-one modifications. To be precise, for every extension $\pi:(X, T) \rightarrow(Y, T)$ between minimal systems there exists a canonically defined commutative diagram of extensions (called the shadow diagram)

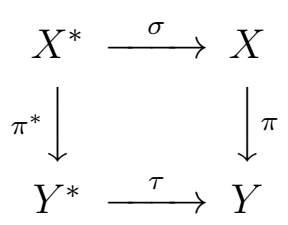

with the following properties:

(1) $\sigma$ and $\tau$ are almost one-to-one extensions;

(2) $\pi^{*}$ is an open extension, i.e. for any open set $U \subset X^{*}, \pi^{*}(U)$ is an open set of $Y^{*}$; 
(3) $X^{*}$ is the unique minimal set in $R_{\pi \tau}=\left\{(x, y) \in X \times Y^{*}: \pi(x)=\tau(y)\right\}$ and $\sigma$ and $\pi^{*}$ are the restrictions to $X^{*}$ of the projections of $X \times Y^{*}$ onto $X$ and $Y^{*}$ respectively.

We refer to [1, 8, 30, 31] for the details of this construction.

In [8] it was shown that, a metric minimal system $(X, T)$ with the property that $n$-proximal tuples are dense in $X^{n}$ for every $n \geq 2$, is weakly mixing. This was extended by van der Woude [33] as follows (see also [9]).

Theorem 4.6. Let $\pi:(X, T) \rightarrow(Y, T)$ be a factor map between the metric minimal systems $(X, T)$ and $(Y, T)$. Suppose that $\pi$ is an open proximal extension, then $\pi$ is a weakly mixing extension, i.e. $\left(R_{\pi}, T \times T\right)$ is transitive.

Now we give the proof of Theorem 4.5,

Proof of Theorem 4.5. Let $(X, T)$ be a minimal system without Ind $_{\text {fip }}$-pairs. We denote by $(Y, T)$ the quotient system of $(X, T)$ determined by the equivalence relation $\mathbf{R P}^{[\infty]}(X, T)$ and let $\pi:(X, T) \rightarrow(Y, T)$ be the canonical projection map.

We first prove that $\pi$ is a proximal extension. Remark that if $(x, y) \in R_{\pi}=\mathbf{R P}^{[\infty]}$ is a $T \times T$ minimal point, according to Corollary 4.4, we have $(x, y)$ is an $\operatorname{Ind}_{\text {fip }}$-pair and thus we must have $x=y$. Now consider any $(x, y) \in R_{\pi}$ and $u \in E(X, T)$ a minimal idempotent. Since $(u x, u y)$ is a $T \times T$ minimal point, we have from previous observation that $u x=u y$, which implies $(x, y)$ is a proximal pair.

As the shadow diagram shows, there exists a canonically defined commutative diagram of extensions

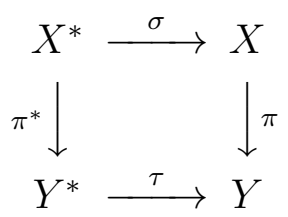

verifying properties (1)-(3) above.

Since $\pi$ is proximal and $\sigma$ is almost one-to-one, we have $\pi \circ \sigma$ is proximal. For any $\left(x, x^{\prime}\right) \in R_{\pi^{*}}, \pi \circ \sigma(x)=\pi \circ \sigma\left(x^{\prime}\right)$, so $\left(x, x^{\prime}\right)$ is a proximal pair, which implies $\pi^{*}$ is proximal too. By Theorem 4.6, $\pi^{*}$ is a weakly mixing extension, and hence there exists $\left(x_{1}, x_{2}\right) \in R_{\pi^{*}}$ such that $R_{\pi^{*}}=\overline{\operatorname{orb}\left(\left(x_{1}, x_{2}\right), T \times T\right)}$. Let $\pi_{1}$ be the projection of $R_{\pi^{*}}$ to the first coordinate. It is easy to get that for any open sets $U, V \subset X^{*}$, $\pi^{*}(U \times V)=\pi^{*}(U) \cap \pi^{*}(V)$ is an open set. So we get that $\pi_{1}$ is an open map too. Since $\left(x_{1}, x_{2}\right)$ is a proximal pair, we have $\left(x_{1}, x_{2}\right)$ is an $\operatorname{Ind}_{\text {fip }}$-pair by Corollary 4.4 . Hence $\left(\sigma\left(x_{1}\right), \sigma\left(x_{2}\right)\right)$ is an $\operatorname{Ind}_{f i p}$-pair too. Then we must have $\sigma\left(x_{1}\right)=\sigma\left(x_{2}\right)$, and thus $R_{\pi^{*}} \subset R_{\sigma}$.

Since $\tau$ is almost one-to-one, we can choose $y \in Y$ such that $\tau^{-1}(y)$ contains only one point. Suppose $x_{1}, x_{2} \in \pi^{-1}(y)$, then there exist $x_{1}^{*}, x_{2}^{*} \in X^{*}$ such that $\sigma\left(x_{1}^{*}\right)=x_{1}$ and $\sigma\left(x_{2}^{*}\right)=x_{2}$. As $\tau \circ \pi^{*}\left(x_{1}^{*}\right)=\pi \circ \sigma\left(x_{1}^{*}\right)=y=\pi \circ \sigma\left(x_{2}^{*}\right)=\tau \circ \pi^{*}\left(x_{2}^{*}\right)$, we have $\pi^{*}\left(x_{1}^{*}\right), \pi^{*}\left(x_{2}^{*}\right) \in \tau^{-1}(y)$ and so $\pi^{*}\left(x_{1}^{*}\right)=\pi^{*}\left(x_{2}^{*}\right)$, i.e. $\left(x_{1}^{*}, x_{2}^{*}\right) \in R_{\pi^{*}} \subset R_{\sigma}$. 
Hence $x_{1}=x_{2}$, which implies that $\pi^{-1}(y)$ contains only one point too. We conclude $\pi$ is almost one-to-one.

Proposition 4.7. Let $(X, T)$ be a minimal distal system. Then there are no non-

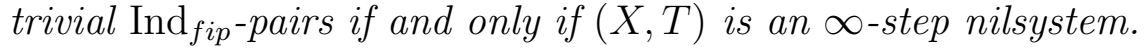

Proof. It is a direct consequence of Theorem 4.5,

4.3. The assumption of semi-openness in Lemma 4.2 cannot be removed. In this subsection we give an example to show that the condition of semi-openness in Lemma 4.2 cannot be removed. First we recall some notions.

Let $(X, T)$ be t.d.s. For any open cover $\mathcal{U}$, let $N(\mathcal{U})$ denote the smallest possible cardinality among finite subcovers of $\mathcal{U}$. Given an increasing sequence $\mathcal{A}=$ $\left\{t_{1}, t_{2}, \ldots\right\}$ in $\mathbb{Z}_{+}$, the sequence entropy of $(X, T)$ or just $T$ with respect to $\mathcal{A}$ and the cover $\mathcal{U}$ is

$$
h_{\mathcal{A}}(T, \mathcal{U})=\limsup _{n \rightarrow \infty} \frac{1}{n} \log N\left(\bigvee_{i=1}^{n} T^{-t_{i}} \mathcal{U}\right)
$$

and the sequence entropy of $T$ with respect to $\mathcal{A}$ is $h_{\mathcal{A}}(T)=\sup _{\mathcal{U}} h_{\mathcal{A}}(T, \mathcal{U})$, where the supremum is taken over all finite open covers $\mathcal{U}$ of $X$. The system $(X, T)$ is a null system if for any sequence $\mathcal{A} \subset \mathbb{Z}_{+}, h_{\mathcal{A}}(T)=0$.

Similar to $\operatorname{Ind}_{\text {fip }}$-pair we can define IN-pairs. Let $(X, T)$ be t.d.s. and $\left(x_{1}, x_{2}\right) \in$ $X \times X$. Then, $\left(x_{1}, x_{2}\right)$ is a IN-pair if for any neighborhoods $U_{1}, U_{2}$ of $x_{1}, x_{2}$ respectively, $\operatorname{Ind}\left(U_{1}, U_{2}\right)$ contains arbitrary long finite independence sets. In [24] is shown that $(X, T)$ is a null system if and only if it contains no nontrivial IN-pairs. It is obvious that a null system contains no nontrivial $\operatorname{Ind}_{f i p}$-pairs as $\operatorname{Ind}_{\text {fip }}$-pairs are IN-pairs.

The following example is classical.

Example 4.8. Sturmian system.

Let $\alpha$ be an irrational number in the interval $(0,1)$ and $R_{\alpha}$ be the irrational rotation on the (complex) unit circle $\mathbb{T}$ generated by $e^{2 \pi i \alpha}$. Set

$$
A_{0}=\left\{e^{2 \pi i \theta}: 0 \leq \theta<(1-\alpha)\right\} \text { and } A_{1}=\left\{e^{2 \pi i \theta}:(1-\alpha) \leq \theta<1\right\}
$$

Consider $z \in \mathbb{T}$ and define $x \in\{0,1\}^{\mathbb{Z}}$ by: for all $n \in \mathbb{Z}, x_{n}=i$ if and only if $R_{\alpha}^{n}(z) \in A_{i}$. Let $X \subset\{0,1\}^{\mathbb{Z}}$ be the orbit closure of $x$ under the shift map $\sigma$ on $\{0,1\}^{\mathbb{Z}}$, i.e. for any $y \in\{0,1\}^{\mathbb{Z}},(\sigma(y))_{n}=y_{n+1}$. This system is called Sturmian system. It is well known that $(X, \sigma)$ is a minimal almost one-to-one extension of $\left(\mathbb{T}, R_{\alpha}\right)$. Moreover, it is an asymptotic extension. Also, it is not hard to prove that it is a null system.

Let $\pi: X \rightarrow \mathbb{T}$ be the former extension and consider $\left(x_{1}, x_{2}\right) \in R_{\pi} \backslash \Delta_{X}$. Then $\left(x_{1}, x_{2}\right)$ is an asymptotic pair and thus $\left(x_{1}, x_{2}\right) \in \mathbf{R} \mathbf{P}^{[d]}$ for any integer $d \geq 1$. In particular, $\left(x_{2}, x_{1}, \ldots, x_{1}\right) \in \mathbf{Q}^{[d+1]}$ for any integer $d \geq 1$. Now, if $\left\{x_{1}, x_{2}\right\}^{[d+1]} \subset$ $\mathbf{Q}^{[d+1]}$ for any integer $d \geq 1$, then, by Lemma 4.1, $\left(x_{1}, x_{2}\right)$ is a nontrivial Ind $_{\text {fip }}$-pair, 
and so an IN-pair, which contradicts the fact that $(X, T)$ is a null system. Therefore, there exists an integer $d \geq 1$ such that $\left\{x_{1}, x_{2}\right\}^{[d]} \nsubseteq \mathbf{Q}^{[d]}$, which implies the condition $\pi_{1}: \overline{\operatorname{orb}((x, y), T \times T)} \rightarrow X$ is semi-open in Lemma 4.2 cannot be removed.

\section{Minimal Distal Systems WhiCH ARE NOT $\infty$-STEP NILSyStemS}

In the previous section we showed that a minimal distal system is an $\infty$-step nilsystem if and only if it has no nontrivial $\operatorname{Ind}_{\text {fip-pairs. In this section we will }}$ give examples of minimal distal systems which are not $\infty$-step nilsystems. We remark that if $(X, T)$ is minimal distal and $\pi:(X, T) \longrightarrow\left(X_{e q}, T\right)$ is the maximal equicontinuous factor of $(X, T)$, then each pair in $R_{\pi} \backslash \Delta_{X}$ is an untame pair (see [20, 17, 27]). In fact, the result in previous section tells that if $\pi_{\infty}:(X, T) \longrightarrow$ $\left(Z_{\infty}, T\right)$ is the factor map from $X$ to its maximal $\infty$-step nilfactor, then each pair in $R_{\pi_{\infty}} \backslash \Delta_{X}$ is an Ind $_{\text {fip }}$-pair. We do not know how to glue both results together.

5.1. The existence. To show the existence of minimal distal systems which are not $\infty$-step nilsystems we use some abstract results from [26]. We use freely notations therein.

Proposition 5.1. [26, Theorem 4.4] Every ergodic measurable distal system $(X, \mathcal{B}, \mu, T)$ can be represented as a minimal topologically distal system equipped with a Borel measure of full support.

Lemma 5.2. [26, Claim 5.5] Suppose $(Y, \mathcal{D}, \nu, T)$ is an isometric extension of the ergodic rotation $\left(\mathbb{T}, \mathcal{B}, \lambda, R_{\alpha}\right)$ by a finite group that is not a Kronecker system. Then $(Y, \mathcal{D}, \nu, T)$ does not have a uniquely ergodic distal model.

The following result produces as much examples as we want.

Theorem 5.3. Suppose $(Y, \mathcal{D}, \nu, T)$ is an isometric extension of the ergodic rotation $\left(\mathbb{T}, \mathcal{B}, \lambda, R_{\alpha}\right)$ by a finite group, that is not a Kronecker system. Then any minimal distal topological model of $(Y, \mathcal{D}, \nu, T)$ is not an $\infty$-step nilsystem.

Proof. By Proposition [5.1, $(Y, \mathcal{D}, \nu, T)$ has a minimal topologically distal system $(X, T)$ model equipped with a Borel measure of full support. By Lemma $5.2,(X, T)$ cannot be uniquely ergodic. It is clear that a minimal $\infty$-step nilsystem is uniquely ergodic, so $(X, T)$ is not an $\infty$-step nilsystem.

5.2. An explicit example. A way to produce an explicit example is to use the following Furstenberg result. It appeared first in [6]. We recall that a topological dynamical system is strictly ergodic if it is minimal and uniquely ergodic.

Theorem 5.4. [6, Theorem 3.1] Let $\left(\Omega_{0}, T_{0}\right)$ be a strictly ergodic system and $\mu_{0}$ its unique ergodic measure. Let $\Omega=\Omega_{0} \times \mathbb{T}$ and let $T: \Omega \rightarrow \Omega$ be defined by $T\left(\omega_{0}, s\right)=\left(T_{0}\left(\omega_{0}\right), g\left(\omega_{0}\right) s\right)$, where $g: \Omega_{0} \rightarrow \mathbb{T}$ is a continuous function. Then, if the equation

$$
g^{k}\left(\omega_{0}\right)=R\left(T_{0}\left(\omega_{0}\right)\right) / R\left(\omega_{0}\right)
$$


has a solution $R: \Omega_{0} \rightarrow \mathbb{T}$ which is measurable but not equal almost everywhere to a continuous function, then $\lim _{N \rightarrow \infty} \frac{1}{N} \sum_{n=0}^{N-1} f \circ T^{n}(\omega)$ cannot exist for all continuous functions $f$ and all $\omega \in \Omega$.

Now we recall some elements of the example from [6] satisfying the criterion of the previous theorem. The first step (that we omit here) is the construction of a sequence of integers $\left(n_{k}\right)_{k \in \mathbb{Z}}$ and an irrational number $\alpha$ such that

$$
h(\theta)=\sum_{k \neq 0} \frac{1}{|k|}\left(e^{2 \pi i n_{k} \alpha}-1\right) e^{2 \pi i n_{k} \theta}
$$

and $g\left(e^{2 \pi i \theta}\right)=e^{2 \pi i \lambda h(\theta)}$, where $\lambda$ is as yet undetermined, are $C^{\infty}$ functions of $[0,1)$ and $\mathbb{T}$ respectively. Clearly, $h(\theta)=H(\theta+\alpha)-H(\theta)$, where

$$
H(\theta)=\sum_{k \neq 0} \frac{1}{|k|} e^{2 \pi i n_{k} \theta} .
$$

Thus $H(\cdot)$ is in $L^{2}(0,1)$ and in particular defines a measurable function. However, $H(\cdot)$ cannot correspond to a continuous function since $\sum_{k \neq 0} \frac{1}{|k|}=\infty$ and hence the series is not Cesàro summable at $\theta=0([35])$. Therefore, for some $\lambda, e^{2 \pi i \lambda H(\theta)}$ cannot be a continuous function either. Considering $R\left(e^{2 \pi i \theta}\right)=e^{2 \pi i \lambda H(\theta)}$ we get

$$
R\left(e^{2 \pi i \alpha} s\right) / R(s)=g(s)
$$

with $R: \mathbb{T} \rightarrow \mathbb{T}$ measurable but not continuous.

By Theorem 5.4, the transformation $T$ of $\mathbb{T} \times \mathbb{T}$ given by

$$
T\left(s_{1}, s_{2}\right)=\left(e^{2 \pi i \alpha} s_{1}, g\left(s_{1}\right) s_{2}\right)
$$

will not possess all its ergodic averages, i.e. there are a continuous function $f$ and $\omega \in \mathbb{T} \times \mathbb{T}$ such that $\lim _{N \rightarrow \infty} \frac{1}{N} \sum_{n=0}^{N-1} f \circ T^{n}(\omega)$ does not exist.

Let $\Omega=\overline{\operatorname{orb}(\omega, T)} \subset \mathbb{T} \times \mathbb{T}$. It is easy to get that $(\mathbb{T} \times \mathbb{T}, T)$ is distal, and so $(\Omega, T)$ is minimal distal. If $(\Omega, T)$ is an $\infty$-step nilsystem, then it is an inverse limit of minimal nilsystems, and of course $(\Omega, T)$ is strictly ergodic. Let $h=\left.f\right|_{\Omega}$ and $h$ apparently continuous on $\Omega$, so $\lim _{N \rightarrow \infty} \frac{1}{N} \sum_{n=0}^{N-1} g \circ T^{n}(\omega)$ exists, contradicting the choice of $f$ and $\omega$. Therefore $(\Omega, T)$ is minimal distal but not an $\infty$-step nilsystem.

\section{Discussion about the unique ERgodicity}

In this section we aim to investigate the question whether a minimal system with-

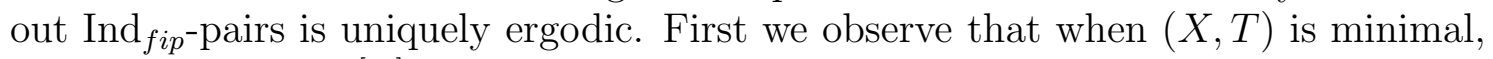
then $\left(X_{\infty}=X / \mathbf{R} \mathbf{P}^{[\infty]}, T\right)$ is uniquely ergodic since it is an inverse limit of uniquely ergodic systems. 
Assume that $(X, T)$ is a minimal system and let $\mu$ be an ergodic measure for $(X, T)$. Then $(X, \mathcal{B}, \mu, T)$ is an ergodic measure preserving system, where $\mathcal{B}$ is the $\sigma$-algebra of Borel sets of $X$ (we omit $\mathcal{B}$ in the sequel). In [15], to prove the convergence in $L^{2}(X, \mathcal{B}, \mu)$ of some non-conventional ergodic averages, the authors introduced measures $\mu^{[d]} \in \mathcal{M}\left(X^{[d]}, T^{[d]}\right)$ for any integer $d \geq 1$ and used them to produce the maximal measure theoretical factor of order $d$ of $(X, \mu, T)$ (in the measurable context this means that the system is an inverse limit, with measurable factor maps, of $d$-step nilsystems), denoted by $\left(Z_{d}, \mathcal{Z}_{d}, \mu_{d}, T\right)$.

In the topological setting, $\left(X_{d}=X / \mathbf{R} \mathbf{P}^{[d]}, T\right)$ is the maximal factor of order $d$ of $(X, T)$ and is uniquely ergodic. In [16] it was observed that $\left(X_{d}, T\right)$ is also a system of order $d$ in the measurable sense for its unique invariant measure. This implies that $X_{d}$ is a factor of $Z_{d}$ in the measurable sense.

Let $\mu=\int_{Z_{d}} \mu_{z} d \mu_{d}(z)$ be the disintegration of $\mu$ over $\mu_{d}$. Pairs in the support of the measure

$$
\lambda_{d}=\int_{Z_{d}} \mu_{z} \times \mu_{z} d \mu_{d}(z)
$$

are called $\mathcal{F}_{d}^{\mu}$-pairs, where $\mathcal{F}_{d}^{\mu}=\operatorname{supp}\left(\lambda_{d}\right)$. To study $\mathcal{F}_{d}^{\mu}$ we will need the following lemma from [15].

Lemma 6.1. [15, Propostion 4.7., Theorem 13.1.] Let $d \geq 1$ be an integer and $V_{d}=\{0,1\}^{[d]}$.

(1) For $f_{\epsilon}, \epsilon \in V_{d}$, bounded measurable functions on $X$,

$$
\int_{X^{[d]}} \bigotimes_{\epsilon \in V_{d}} f_{\epsilon} d \mu^{[d]}=\int_{Z_{d-1}^{[d]}} \bigotimes_{\epsilon \in V_{d}} \mathbb{E}\left(f_{\epsilon} \mid \mathcal{Z}_{d-1}\right) d \mu_{d-1}^{[d]},
$$

where $\left(Z_{d-1}^{[d]}, \mu_{d-1}^{[d]}\right)$ is the joining of $2^{d}$ copies of $\left(Z_{d-1}, \mu_{d-1}\right)$.

(2) For $f_{\epsilon}, \epsilon \in V_{d}$, bounded measurable functions on $X$, the average

$$
\prod_{i=1}^{d} \frac{1}{N_{i}-M_{i}} \sum_{\mathbf{n} \in\left[M_{1}, N_{1}\right) \times \cdots \times\left[M_{d}, N_{d}\right)} \int_{X} \prod_{\epsilon \in V_{d}} f_{\epsilon} \circ T^{\mathbf{n} \cdot \epsilon} d \mu
$$

converges to

$$
\int_{X^{[d]}} \prod_{\epsilon \in V_{d}} f_{\epsilon}\left(x_{\epsilon}\right) d \mu^{[d]}(\mathbf{x})
$$

as $N_{i}-M_{i} \rightarrow \infty$ for all $1 \leq i \leq d$.

We get the following theorem about $\mathcal{F}_{d}^{\mu}$.

Theorem 6.2. Let $(X, T)$ be a minimal system and $\mu$ an ergodic measure on $X$.

(1) Let $d \geq 1$ be an integer, then $\mathcal{F}_{d}^{\mu} \subset \mathbf{R} \mathbf{P}^{[d]}$.

(2) $\bigcap_{d \in \mathbb{N}} \mathcal{F}_{d}^{\mu} \subset \operatorname{Ind}_{\text {fip }}(X, T)$. 
Proof. Let $\left(x_{0}, x_{1}\right) \in \mathcal{F}_{d}^{\mu}$. Then for any neighborhood $U_{0} \times U_{1}$ of $\left(x_{0}, x_{1}\right)$

$$
\lambda_{d}\left(U_{0} \times U_{1}\right)=\int_{Z_{d}} \mathbb{E}\left(1_{U_{0}} \mid \mathcal{Z}_{d}\right) \mathbb{E}\left(1_{U_{1}} \mid \mathcal{Z}_{d}\right) d \mu_{d}>0
$$

Let $\left\{U_{\epsilon}: \epsilon \in V_{d+1}\right\} \subset\left\{U_{0}, U_{1}\right\}$ be open sets. We claim that

$$
\int_{X^{[d+1]}} \prod_{\epsilon \in V_{d+1}} 1_{U_{\epsilon}}\left(x_{\epsilon}\right) d \mu^{[d+1]}(\mathbf{x})>0 .
$$

Proof of the claim: Since $\int_{Z_{d}} \mathbb{E}\left(1_{U_{0}} \mid \mathcal{Z}_{d}\right) \mathbb{E}\left(1_{U_{1}} \mid \mathcal{Z}_{d}\right) d \mu_{d}>0$, there exists $B \in \mathcal{Z}_{d}$ with $\mu_{d}(B)>0$ such that for any $z \in B, \mathbb{E}\left(1_{U_{0}} \mid \mathcal{Z}_{d}\right)(z) \mathbb{E}\left(1_{U_{1}} \mid \mathcal{Z}_{d}\right)(z)>0$. Also, since $U_{\epsilon}=$ $U_{0}$ or $U_{\epsilon}=U_{1}$ for $\epsilon \in V_{d+1}$, we have for any $\mathbf{z} \in B^{[d+1]}$ that $\prod_{\epsilon \in V_{d+1}} \mathbb{E}\left(1_{U_{\epsilon}} \mid \mathcal{Z}_{d}\right)\left(z_{\epsilon}\right)>$ 0 . Now, by the previous lemma, we obtain

$$
\begin{aligned}
\int_{X^{[d+1]}} \prod_{\epsilon \in V_{d+1}} 1_{U_{\epsilon}}\left(x_{\epsilon}\right) d \mu^{[d+1]}(\mathbf{x}) & =\int_{Z_{d}^{[d+1]}} \prod_{\epsilon \in V_{d+1}} \mathbb{E}\left(1_{U_{\epsilon}} \mid \mathcal{Z}_{d}\right)\left(z_{\epsilon}\right) d \mu_{d}^{[d+1]}(\mathbf{z}) \\
& \geq \int_{B^{[d+1]}} \prod_{\epsilon \in V_{d+1}} \mathbb{E}\left(1_{U_{\epsilon}} \mid \mathcal{Z}_{d}\right)\left(z_{\epsilon}\right) d \mu_{d}^{[d+1]}(\mathbf{z})>0
\end{aligned}
$$

This completes the proof of the claim since it was shown in [15] that $\mu_{d}^{[d+1]}\left(B^{[d+1]}\right) \geq$ $\mu_{d}(B)^{2^{d+1}}>0$.

Again, by Lemma 6.1,

$$
\frac{1}{N^{d+1}} \sum_{0 \leq n_{1}, \ldots, n_{d+1}<N} \int_{X} \prod_{\epsilon \in V_{d+1}} 1_{U_{\epsilon}} \circ T^{n_{1} \epsilon_{1}+\ldots+n_{d+1} \epsilon_{d+1}} d \mu
$$

converges to

$$
\int_{X^{[d+1]}} \prod_{\epsilon \in V_{d+1}} 1_{U_{\epsilon}}\left(x_{\epsilon}\right) d \mu^{[d+1]}(\mathbf{x})>0
$$

as $N \rightarrow \infty$. Hence, there exists a Borel set $B$ with $\mu(B)>0$ and $n_{1}, \ldots, n_{d+1} \in \mathbb{Z}_{+}$ such that for each $x \in B$

$$
\prod_{\epsilon \in V_{d+1}} 1_{U_{\epsilon}} \circ T^{n_{1} \epsilon_{1}+\ldots+n_{d+1} \epsilon_{d+1}}(x)>0 .
$$

That is, for each $x \in B, T^{n_{1} \epsilon_{1}+\ldots+n_{d+1} \epsilon_{d+1}}(x) \in U_{\epsilon}$ or

$$
\bigcap_{\epsilon \in V_{d+1}} T^{-n_{1} \epsilon_{1}-\ldots-n_{d+1} \epsilon_{d+1}} U_{\epsilon} \neq \emptyset \text {. }
$$

First we prove statement (1). Let $\left(x_{0}, x_{1}\right) \in \mathcal{F}_{d}^{\mu}$. For $\delta>0$ let $U_{0} \times U_{1}$ be a neighborhood of $\left(x_{0}, x_{1}\right)$ where the diameters of $U_{0}$ and $U_{1}$ are less than $\delta$. Set $U_{(0, \ldots, 0)}=U_{0}, U_{(0, \ldots, 0,1)}=U_{1}$ and $U_{\epsilon}=U_{0}$ for any other $\epsilon \in V_{d+1}$. By previous discussion, there exist $x \in X$ and $n_{1}, \ldots, n_{d+1} \in \mathbb{Z}_{+}$such that $T^{n_{1} \epsilon_{1}+\ldots+n_{d+1} \epsilon_{d+1}}(x) \in U_{\epsilon}$ for any $\epsilon \in V_{d+1}$. Let $y_{0}=x=T^{0} x \in U_{(0, \ldots, 0)}=U_{0}, y_{1}=T^{n_{d+1}} x \in U_{(0, \ldots, 0,1)}=U_{1}$. Then, for any $\epsilon \in V_{d} \backslash\{(0, \ldots, 0)\}$, we have $T^{n_{1} \epsilon_{1}+\ldots+n_{d} \epsilon_{d}}\left(y_{0}\right) \in U_{\epsilon 0}=U_{0}$ and 
$T^{n_{1} \epsilon_{1}+\ldots+n_{d} \epsilon_{d}}\left(y_{1}\right) \in U_{\epsilon 1}=U_{0}$. Since the diameters of $U_{0}$ and $U_{1}$ are less than $\delta$, we get that $d\left(x_{0}, y_{0}\right)<\delta, d\left(x_{1}, y_{1}\right)<\delta$ and for any $\epsilon \in V_{d} \backslash\{(0, \ldots, 0)\}$, $d\left(T^{n_{1} \epsilon_{1}+\ldots+n_{d} \epsilon_{d}}\left(y_{0}\right), T^{n_{1} \epsilon_{1}+\ldots+n_{d} \epsilon_{d}}\left(y_{1}\right)\right)<\delta$. Therefore $\mathcal{F}_{d}^{\mu} \subset \mathbf{R P}^{[d]}$.

To show (2), by Lemma 4.1, it remains to prove that if $\left(x_{0}, x_{1}\right) \in \bigcap_{d \in \mathbb{N}} \mathcal{F}_{d}^{\mu}$ then $\left\{x_{0}, x_{1}\right\}^{[d]} \subset \mathbf{Q}^{[d]}$ for any integer $d \geq 1$. Let $d \geq 1$ be an integer and take $\mathbf{x}=$ $\left(x_{\epsilon}\right)_{\epsilon \in V_{d}} \in\left\{x_{0}, x_{1}\right\}^{[d]}$. Given a neighborhood $\mathbf{V}$ of $\mathbf{x}$, there exists a neighborhood $U_{0} \times U_{1}$ of $\left(x_{0}, x_{1}\right)$ such that if we set $U_{\epsilon}=U_{i}$ depending on $x_{\epsilon}=x_{0}$ or $x_{\epsilon}=x_{1}$, then $\bigotimes_{\epsilon \in V_{d}} U_{\epsilon} \subset \mathbf{V}$. From the conclusion of part (1), there exist $x \in X$ and $n_{1}, \ldots, n_{d} \in \mathbb{Z}_{+}$such that $T^{n_{1} \epsilon_{1}+\ldots+n_{d} \epsilon_{d}}(x) \in U_{\epsilon}$ for any $\epsilon \in V_{d}$. Let $\mathbf{n}=\left(n_{1}, \ldots, n_{d}\right)$. We have $\left(T^{\mathbf{n} \cdot \epsilon} x\right)_{\epsilon \in V_{d}} \in \mathbf{V}$, and thus $\mathbf{x} \in \mathbf{Q}^{[d]}$. The proof is completed.

Remark 6.3. We have shown that $\mathcal{F}_{d}^{\mu} \subset \mathbf{R P}^{[d]}$. However, the converse is not true in general. For example, let $(Z, S)$ be a non-trivial $d$-step nilsystem and $\nu$ an ergodic measure on $Z$. In [32] it was shown that there exists a weakly mixing minimal uniquely ergodic system $(X, T)$ with the uniquely ergodic measure $\mu$ satisfying that for all $x, y \in X$ with $y \notin\left\{T^{n} x\right\}_{n \in \mathbb{Z}}$, the orbit $\left\{\left(T^{n} x, T^{n} y\right)\right\}_{n \in \mathbb{Z}}$ is dense in $X \times X$; and $(Z, \nu, S)$ and $(X, \mu, T)$ are isomorphic. Then $(X, \mu)$ coincides with $\left(Z_{d}(X), \mu_{d}\right)$, and so $\mathcal{F}_{\mu}^{d}=\Delta_{X}$. Since $(X, T)$ is weakly mixing, we get that $\mathbf{R P}^{[d]}=X \times X$, which implies that $\mathcal{F}_{\mu}^{d} \varsubsetneqq \mathbf{R P}^{[d]}$.

A direct application of the above theorem is the following.

Theorem 6.4. Let $(X, T)$ be a minimal system with $\operatorname{Ind}_{\text {fip }}(X, T)=\Delta_{X}$, then for each ergodic measure $\mu,(X, \mu, T)$ is measure theoretical isomorphic to an $\infty$-step nilsystem.

Proof. Applying Theorem 6.2 we get that $\bigcap_{d \in \mathbb{N}} \mathcal{F}_{d}^{\mu} \subset \operatorname{Ind}_{\text {fip }}(X, T)=\Delta_{X}$. The result follows, since it is easy to check that $\bigcap_{d \in \mathbb{N}} \mathcal{F}_{d}^{\mu}=\mathcal{F}_{\infty}^{\mu}$, where $\mathcal{F}_{\infty}^{\mu}$ is the support of $\lambda_{\infty}=\int_{\mathcal{Z}_{\infty}} \mu_{z} \times \mu_{z} d \mu_{\infty}(z)$ with $\left(Z_{\infty}, \mu_{\infty}\right)$ the inverse limit of $\left(Z_{d}, \mu_{d}\right)$.

To show the unique ergodicity of an $\infty$-step nilsystem the following question is crucial.

Question 6.5. Let $(X, T)$ be an E-system (i.e. is transitive and admits an invariant measure with full support), let $x$ be a transitive point and $p$ be a fixed point of $(X, T)$. Is it true that $(p, x, \ldots, x) \in \mathbf{Q}^{[d]}$ for any integer $d \geq 1$ ?

If this question has a positive answer, then by Lemma 4.2 , we have $\{x, p\}^{[d]} \subset \mathrm{Q}^{[d]}$ for any integer $d \geq 1$ ( since $\overline{\operatorname{orb}((x, p), T \times T)}=X \times\{p\})$, and thus $(x, p)$ is an

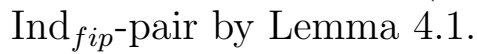

Conjecture 6.6. Let $(X, T)$ be a minimal system with $\operatorname{Ind}_{\text {fip }}(X, T)=\Delta_{X}$. Then $(X, T)$ is uniquely ergodic.

If Question 6.5 has a positive answer, then using the proof of [20, Theorem 4.4] and the lifting property of $\operatorname{Ind}_{\text {fip }}$-pairs [19], we may conclude that the conjecture holds. 


\section{Topological COMPlEXity OF $\infty$-STEP NILSYSTEMS}

The big development in the study of non-conventional ergodic averages during the last decade has put in evidence, among other facts, the crucial role of nilsystems when studying "polynomial" phenomena in dynamical systems theory. The objective of this section is to prove that $\infty$-step nilsystems have polynomial topological complexity. It is well known that bounded complexity characterize minimal rotations on compact Abelian groups (see [4]). Some basic symbolic examples (substitutions systems for example) show that polynomial complexity cannot characterize $\infty$-step nilsystems, so the characterizion of polynomial complexity seems to be a deep problem far to be solved.

In order to study the quantitative distribution of polynomial orbits in nilmanifolds, Green and Tao introduced in [13] a metric induced by the Mal'cev basis on a nilmanifold and they studied its behaviour under left multiplication. We obtain as an application a polynomial bound for the topological complexity of nilsystems and consequently of $\infty$-step nilsystems. We use freely the notations and results from [13] and [2].

7.1. Polynomial behaviour of orbits. In this subsection we assume $G$ is a connected and simply connected Lie Group and $\Gamma \subset G$ is a co-compact subgroup. We will denote by $G_{i}$ the $i$-th subgroup of the associated lower central series. Under these assumptions Mal'cev proved the following theorem.

Theorem 7.1 (Mal'cev basis). Let $G$ be an m-dimensional nilpotent Lie group and $\Gamma \subset G$ a co-compact subgroup. There exists a basis $\mathcal{X}=\left\{X_{1}, \ldots, X_{m}\right\}$ of the associated Lie algebra $\mathfrak{g}$ such that:

(1) For each $j \in\{0, \ldots, m-1\}$ the subspace $\mathfrak{h}_{j}=\operatorname{Span}\left(X_{j+1}, \ldots, X_{m}\right)$ is an ideal in $\mathfrak{g}$ and $\exp \left(\mathfrak{h}_{j}\right)$ is a normal subgroup in $G$.

(2) $G_{i}=\exp \left(\mathfrak{h}_{m-m_{i}}\right)$, where $m_{i}=\operatorname{dim}\left(G_{i}\right)$.

(3) Each $g \in G$ can be written uniquely as $\exp \left(t_{1} X_{1}\right) \cdots \exp \left(t_{m} X_{m}\right)$ where $t=$ $\left(t_{1}, \ldots, t_{m}\right) \in \mathbb{R}^{m}$

(4) $\Gamma=\left\{\exp \left(n_{1} X_{1}\right) \cdots \exp \left(n_{m} X_{m}\right): n=\left(n_{1}, \ldots, n_{m}\right) \in \mathbb{Z}^{m}\right\}$.

We say that $\mathcal{X}=\left\{X_{1}, \ldots, X_{m}\right\}$ is a Mal'cev basis for $G / \Gamma$ adapted to the lower central series $\left(G_{i}\right)_{i \geq 0}$.

Let $g=\exp \left(t_{1} X_{1}\right) \cdots \exp \left(t_{m} X_{m}\right) \in G$ and denote $\psi(g)=\left(t_{1}, \ldots, t_{m}\right) \in \mathbb{R}^{m}$. Let $|\psi(g)|=\|\psi(g)\|_{\infty}$. Fix a Mal'cev basis $\mathcal{X}$. In [13], the authors introduced the following metric on $G$ and $G / \Gamma$.

Definition 7.2. Let $x, y \in G$ and define

$d(x, y)=\inf \left\{\sum_{i=0}^{n-1} \min \left(\left|\psi\left(x_{i-1} x_{i}^{-1}\right)\right|,\left|\psi\left(x_{i} x_{i-1}^{-1}\right)\right|\right): n \in \mathbb{N}, x_{0}, \ldots, x_{n} \in G, x_{0}=x, x_{n}=y\right\}$

which is right-invariant, i.e. $d(x, y)=d(x g, y g)$ for all $g \in G$ and $d(x, y) \leq$ $\left|\psi\left(x y^{-1}\right)\right|$. 
This metric induces a metric on $G / \Gamma$ that we also call $d(\cdot, \cdot)$ by setting:

$$
\begin{aligned}
d(x \Gamma, y \Gamma) & =\inf \left\{d\left(x^{\prime}, y^{\prime}\right): x^{\prime} \in x \Gamma, y^{\prime} \in y \Gamma\right\} \\
& =\inf \left\{d\left(x \gamma_{1}, y \gamma_{2}\right): \gamma_{1}, \gamma_{2} \in \Gamma\right\}=\inf \{d(x, y \gamma): \gamma \in \Gamma\}
\end{aligned}
$$

where the last equality follows from the right-invariance of the metric.

In the sequel we use some results obtained in [13. and we rephrase some others in a convenient way. The Mal'cev basis $\mathcal{X}$ is fixed.

Lemma 7.3 (Multiplication and inversion). Let $x, y \in G, t=\psi(x), u=\psi(y)$. Then,

(1) $\psi(x y)=\left(t_{1}+u_{1}, t_{2}+u_{2}+P_{1}\left(t_{1}, u_{1}\right), \ldots, t_{m}+u_{m}+P_{m-1}\left(t_{1}, \ldots, t_{m-1}, u_{1}, \ldots, u_{m-1}\right)\right)$ where for each $i \in\{1, \ldots, m-1\}, P_{i}$ is a real polynomial.

(2) $\psi\left(x^{-1}\right)=\left(-t_{1},-t_{2}+Q_{1}\left(t_{1}\right), \ldots,-t_{m}+Q_{m-1}\left(t_{1}, \ldots, t_{m-1}\right)\right)$ where for each $i \in\{1, \ldots, m\}, Q_{i}$ is a real polynomial.

We get easily that $\psi\left(x y^{-1}\right)=\left(R_{1}(t, u), R_{2}(t, u), \ldots, R_{m}(t, u)\right)$ where $R_{i}$ is a real polynomial for each $i \in\{1, \ldots, m\}$. In order to simplify notations in what follows we will write $P$ to refer to any generic real polynomial with positive coefficients, not necessarily the same. This will be clear from the context.

Lemma 7.4 (Coordinates polynomial bound). Let $x, y \in G$. Then,

$$
d(x, y) \leq P(\max (|\psi(x)|,|\psi(y)|))|\psi(x)-\psi(y)|
$$

Proof. Let $\psi(x)=t$ and $\psi(y)=u$. From the definition of the distance and Lemma 7.3 we have,

$$
d(x, y) \leq\left|\psi\left(x y^{-1}\right)\right|=\left|\left(R_{1}(t, u), R_{2}(t, u), \ldots, R_{m}(t, u)\right)\right| .
$$

Write $R_{i}(t, u)=\sum_{\vec{\alpha}, \vec{\beta}} C_{\vec{\alpha}, \vec{\beta}}^{(i)} t^{\vec{\alpha}} u^{\vec{\beta}}$. Since $R_{i}(t, t)=0$, one deduces

$$
R_{i}(t, u)=R_{i}(t, u)-R_{i}(t, t)=\sum_{\vec{\alpha}, \vec{\beta}} C_{\vec{\alpha}, \vec{\beta}}^{(i)} t^{\vec{\alpha}}\left(u^{\vec{\beta}}-t^{\vec{\beta}}\right)
$$

Expanding $\left(u^{\vec{\beta}}-t^{\vec{\beta}}\right)=\sum_{i=1}^{m}\left(u_{i}-t_{i}\right) W_{\vec{\beta}, i}(t, u)$, where $W_{\vec{\beta}, i}$ are polynomials, we get, $d(x, y) \leq|\psi(x)-\psi(y)| \sum_{\vec{\alpha}, \vec{\beta}} \sum_{i=1}^{m}\left|C_{\vec{\alpha}, \vec{\beta}}^{(i)}\right| t^{\vec{\alpha}}|| W_{\vec{\beta}, i}(t, u)|\leq| \psi(x)-\psi(y) \mid P(\max (|\psi(x)|,|\psi(y)|))$.

Lemma 7.5. Let $g, x, y \in G$, then

$$
d(g x, g y) \leq P(|\psi(g)|,|\psi(x)|,|\psi(y)|) d(x, y)
$$


Proof. Let $g, z \in G$. From Lemma 7.3 we see that $\psi\left(g z g^{-1}\right)$ is a polynomial function of $\psi(z)$ and $\psi(g)$ that vanishes when $\psi(z)=0$. This this polynomial function can be written as $\psi(z) P(\psi(g), \psi(z))$. Then,

$$
\left|\psi\left(g z g^{-1}\right)\right| \leq|\psi(z)| P(|\psi(z)|,|\psi(g)|)
$$

By Lemma 7.4, $d(x, y) \leq P(|\psi(x)|,|\psi(y)|)$, and then for computing the distance between $x$ and $y$ we can restrict to paths $x=x_{0}, \ldots, x_{n}=y$ satisfying $k\left(x_{i}, x_{i+1}\right) \leq$ $P(|\psi(x)|,|\psi(y)|)$ where $k\left(x_{i}, x_{i+1}\right)=\min \left(\left|\psi\left(x_{i} x_{i+1}^{-1}\right)\right|,\left|\psi\left(x_{i+1} x_{i}^{-1}\right)\right|\right)$. Let us observe (using Lemma 7.3) that this property implies that $\max \left(\left|\psi\left(x_{i} x_{i+1}^{-1}\right)\right|,\left|\psi\left(x_{i+1} x_{i}^{-1}\right)\right|\right) \leq$ $P(|\psi(x)|,|\psi(y)|)$.

Consider $x=x_{0}, \ldots, x_{n}=y$ such a path and the path $g x=g x_{0}, g x_{1}, \ldots, g x_{n}=$ gy. From (7.1),

$$
\begin{aligned}
k\left(g x_{i}, g x_{i+1}\right) & \leq\left|\psi\left(g x_{i} x_{i+1}^{-1} g^{-1}\right)\right| \\
& \leq\left|\psi\left(x_{i} x_{i+1}^{-1}\right)\right| P\left(\left|\psi\left(x_{i} x_{i+1}^{-1}\right)\right|,|\psi(g)|\right) \\
& \leq\left|\psi\left(x_{i} x_{i+1}^{-1}\right)\right| P(|\psi(x)|,|\psi(y)|,|\psi(g)|)
\end{aligned}
$$

In the same way,

$$
\left|\psi\left(g x_{i+1} x_{i}^{-1} g^{-1}\right)\right| \leq\left|\psi\left(x_{i+1} x_{i}^{-1}\right)\right| P(|\psi(x)|,|\psi(y)|,|\psi(g)|)
$$

and then

We conclude,

$$
k\left(g x_{i}, g x_{i+1}\right) \leq P(|\psi(x)|,|\psi(y)|,|\psi(g)|)\left|k\left(x_{i}, x_{i+1}\right)\right|
$$

$$
\begin{aligned}
d(g x, g y) & \leq k\left(g x_{0}, g x_{1}\right)+\ldots+k\left(g x_{n-1}, g x_{n}\right) \\
& \leq P(|\psi(x)|,|\psi(y)|,|\psi(g)|)\left(k\left(x_{0}, x_{1}\right)+\ldots+k\left(x_{n-1}, x_{n}\right)\right)
\end{aligned}
$$

and the lemma follows taking the infimum.

Lemma 7.6. Let $g \in G$ and $n \in \mathbb{N}$, then $\left|\psi\left(g^{n}\right)\right| \leq P(n)$, where $P$ is a polynomial with coefficients depending on $|\psi(g)|$.

Proof. By the multiplication formula we observe that $\left(\psi\left(g^{n}\right)\right)_{1}=n \psi(g)_{1}$, i.e. the first coordinate is controlled polynomially. Suppose now that the $i$-th coordinate is controlled polynomially, then the same happens with the $i+1$-th coordinate. In fact, we see inductively that,

$\psi\left(g^{n+1}\right)_{i+1}=\psi\left(g^{n} g\right)_{i+1}=\psi\left(g^{n}\right)_{i+1}+\psi(g)_{i+1}+P\left(\psi\left(g^{n}\right)_{1}, \ldots, \psi\left(g^{n}\right)_{i}, \psi(g)_{1}, \ldots, \psi(g)_{i}\right)$ and then $\left|\psi\left(g^{n+1}\right)_{i+1}\right|-\left|\psi\left(g^{n}\right)_{i+1}\right| \leq P(n)$, and we conclude $\left|\psi\left(g^{n+1}\right)_{i+1}\right| \leq(n+$ 1) $P(n+1)$. (Here all polynomials $P$ are not necessarily the same.)

Lemma 7.7 (Factorization). Each $g \in G$ can be written in a unique way as $g=$ $\{g\}[g]$ with $\psi(\{g\}) \in[0,1)^{m}$ and $[g] \in G$.

Therefore, when writing $x \Gamma$ we can assume $x$ is such that $|\psi(x)| \leq 1$. 
Lemma 7.8. Let $x, y \in G$. Then,

$$
d(x \Gamma, y \Gamma)=\inf _{\gamma \in \Gamma} d(x, y \gamma)=\inf _{\gamma \in \Gamma,|\psi(\gamma)| \leq C} d(x, y \gamma)
$$

where $C$ is a constant depending only on $|\psi(x)|$ and $|\psi(y)|$.

Combining the last two lemmas we see that:

$$
d(x \Gamma, y \Gamma)=\inf _{\gamma \in \Gamma,|\psi(\gamma)| \leq C} d(x, y \gamma)
$$

where $C$ is a constant.

We obtain,

$$
d\left(g^{n} x \Gamma, g^{n} y \Gamma\right) \leq \inf _{\gamma \in \Gamma,|\psi(\gamma)| \leq C} d\left(g^{n} x, g^{n} y \gamma\right)
$$

Using Lemmas 7.5, 7.6 and 7.8 we get,

Corollary 7.9. Let $x, y, g \in G$ with $|\psi(x)| \leq 1$ and $|\psi(y)| \leq 1$. Then,

$$
d\left(g^{n} x \Gamma, g^{n} y \Gamma\right) \leq \inf _{\gamma \in \Gamma,|\psi(\gamma)| \leq C} P\left(\left|\psi\left(g^{n}\right)\right|\right) d(x, y \gamma) \leq P(n) d(x \Gamma, y \Gamma)
$$

7.2. Complexity computation. Let $(X, T)$ be a t.d.s. We say that a subset $F \subseteq$ $X$ is $(n, \epsilon)$-shadowing if for all $x \in X$ there exists $y \in F$ such that $d\left(T^{i} x, T^{i} y\right) \leq \epsilon$ for all $i \in\{0, \ldots, n\}$. Write $r(n, \epsilon)=\min \{|F|: F \subseteq X, F$ is $(n, \epsilon)$-shadowing $\}$. Given an open cover $\mathcal{U}=\left\{U_{1}, \ldots, U_{k}\right\}$ the topological complexity function of $\mathcal{U}$ is the sequence on $n: c(\mathcal{U}, n)=\min \left\{M \geq 1: \exists V_{1}, \ldots, V_{M} \in \bigvee_{i=0}^{n} T^{-i} \mathcal{U}\right.$, such that $\bigcup_{i=1}^{M} V_{i}=$ $X\}$. One has that,

$$
c(\mathcal{U}, n) \leq r\left(n, \frac{\delta}{2}\right)
$$

where $\delta>0$ is the Lebesgue number of $\mathcal{U}$.

To compute the topological complexity of a nilsystem in the general case (i.e. when $G$ is not neccesarily a connected and simply connected Lie group) we will use an argument given by A.Leibman in [25]. For that we require an extra definition and one lemma from [25].

Let $X=G / \Gamma$ be a nilmanifold and $T: X \rightarrow X$ the transformation given by $x \rightarrow g x$ with a fixed $g \in G$.

Definition 7.10. We say that a closed subset $Y \subset X$ is a submanifold of $X$ if $Y=H x$ where $H$ is a closed subgroup of $G$ and $x \in X$.

Lemma 7.11. There exists a connected, simply connected nilpotent Lie group $\widehat{G}$ and $\widehat{\Gamma} \subseteq \widehat{G}$ a co-compact subgroup such that $X$ with the action of $G$ is isomorphic to a submanifold $\widetilde{X}$ of $\widehat{X}=\widehat{G} / \widehat{\Gamma}$ representing the action of $G$ in $\widehat{G}$.

This is the main result of the section. 
Theorem 7.12. Let $X=G / \Gamma$ be a nilmanifold and $T: X \rightarrow X$ the transformation given by $x \rightarrow g x$ with a fixed $g \in G$. Let $\mathcal{U}$ be an open cover of $X$. Then, for all $n \in \mathbb{N}$,

where $P$ is a polynomial.

$$
c(\mathcal{U}, n) \leq P(n)
$$

Proof. First, assume that $G$ is a connected, simply connected nilpotent Lie Group. For $\epsilon>0$, let $N(\epsilon)$ be the smallest number of balls of ratio $\epsilon$ needed to cover $X$. The upper Minkowski dimension or box dimension (see [28]) is defined by

$$
\limsup _{\epsilon \rightarrow 0} \frac{\log N(\epsilon)}{\log (1 / \epsilon)}
$$

This dimension coincides with the usual dimension of the manifold $X$ and hence there exists a constant $K$ such that:

$$
N(\epsilon) \leq K\left(\frac{1}{\epsilon}\right)^{\operatorname{dim}(X)+1}
$$

Using the bound in (7.2) we observe that if $x, y \in X$ and $d(x, y) \leq \frac{\delta}{2 P(n)}$, then $d\left(T^{i} x, T^{i} y\right) \leq \frac{P(i)}{P(n)} \frac{\delta}{2} \leq \frac{\delta}{2}$ if $i \leq n$ since $P$ has positive coefficients. Let $\delta$ the Lebesgue number of $\mathcal{U}$. We get,

$$
c(\mathcal{U}, n) \leq N\left(\frac{\delta}{2 P(n)}\right) \leq K \frac{(2 P(n))^{\operatorname{dim}(X)+1}}{\delta^{\operatorname{dim}(X)+1}}
$$

and the polynomial bound of the complexity is obtained.

Now consider the general case. Denote by $\pi: X \rightarrow \widetilde{X}$ the isomorphism given by Lemma 7.11. We see that $(X, T)$ is conjugate with $(\widetilde{X}, \widetilde{T})$ where $\widetilde{T}: \widehat{X} \rightarrow \widehat{X}$ is defined by $\widetilde{T}(\widehat{x})=\pi(g) \widehat{x}$. Hence $(\widetilde{X}, \widetilde{T})$ is a subsystem of $(\widehat{X}, \widetilde{T})$.

If $\mathcal{U}=\left(U_{1}, \ldots, U_{m}\right)$ is an open cover of $X, \pi(\mathcal{U})=\left(\pi\left(U_{1}\right), \ldots, \pi\left(U_{m}\right)\right)$ is an open cover of $\widetilde{X}$ and $c(\mathcal{U}, n)=c(\pi(\mathcal{U}), n) \leq c(\widehat{\mathcal{U}}, n)$ where $\widehat{\pi}(U)=\left(V_{1}, \ldots, V_{m}, \widetilde{X}^{c}\right)$ is an open cover of $\widehat{X}$ with $\pi\left(U_{i}\right)=\widetilde{X} \cap V_{i}$ for all $i \in\{1, \ldots, m\}$. Thus, we get a polynomial bound of the complexity in the general case.

Finally, we consider the complexity of a $\infty$-step nilsystem. For that we need the following easy lemma.

Lemma 7.13. Suppose $X$ is an inverse limit of the systems $\left(X_{i}, T\right)_{i \in \mathbb{N}}$ where $\left(X_{i}, T\right)$ has a polynomial complexity for each $i \in \mathbb{N}$. Then $X$ has polynomial complexity.

Proof. We will show that the product system has polynomial complexity and therefore the inverse limit has the same property. Let $\epsilon>0$ and choose $N \in \mathbb{N}$ such that $\delta=\epsilon-2^{-N}>0$. Then $r_{X}(\epsilon, n) \leq \prod_{i \leq N} r_{X_{i}}(\delta, n)$ and by assumption the right side is polynomially bounded.

We conclude, 
Theorem 7.14. If $X$ is an $\infty$-step nilsystem then it has a polynomial complexity.

Proof. By the above discussion a $d$-step nilsystem has polynomial complexity. By Theorem [16] the factors $\left(X_{d}, T\right)$ defined by the relation $\mathbf{R} \mathbf{P}^{[d]}$ are inverse limits of $d$-step nilsystems and therefore they have polynomial complexity. Using again the inverse limit argument we conclude the polynomial bound for the complexity of $(X, T)$.

\section{ApPENDIX}

In this appendix we give the proof of Theorem 3.8. First we discuss Theorem 3.8 (2). The idea to prove this fact was inspired from personal communications with B. Kra [23], here we give details of the proof.

Lemma 8.1. [14] Let $(X, \mu, T)$ be an ergodic d-step nilsystem with $X=G / \Gamma, \mu$ be its Haar probability measure and $T$ be the translation by the element $t \in G$. Moreover, assume that the group $G$ can be spanned by the connected component of the identity and the element $t$ (it is always possible to reduce to this case, see [3]). Let $d \geq 1$ be an integer. If $Z_{k}$ is the maximal factor of order $d$ of $(X, \mu, T)$ with $k \leq d$, then $Z_{k}$ has the form $G /\left(G_{k+1} \Gamma\right)$ endowed with the translation by the projection of $t$ on $G / G_{k+1}$, where $G_{1}=G, G_{2}=\left[G, G_{1}\right], G_{3}=\left[G, G_{2}\right], \ldots, G_{d+1}=\{e\}$.

Now we prove Theorem 3.8 (2):

Proof of Theorem 3.8 (2): Let $n>d$ be any integer, we will show $Z_{n}=Z_{d}$. In [15] it was shown that $Z_{n}$ is an inverse limit of $n$-step nilsystems $\left(Z_{n, i}\right)_{i \in \mathbb{N}}$. For any $Z_{n, i}$, assume it has the form of $G / \Gamma$, where the group $G$ is spanned by the connected component of the identity and the translation element $t$.

Let $G^{o}$ be the identity component of $G$. Just as showed in $[3], G_{2}=[G, G]=$ $\left[G^{o}, G\right]$ is connected; and inductively for any integer $k \geq 2, G_{k}$ is connected. By Lemma 8.1, the $d$-step maximal nilfactor and $d+1$-step maximal nilfactor of $G / \Gamma$ is $G /\left(G_{d+1} \Gamma\right)$ and $G /\left(G_{d+2} \Gamma\right)$ respectively. We have that $G /\left(G_{d+2} \Gamma\right)$ is also a $d+1$ step nilfactor of $X$, so it is a factor of $Z_{d+1}=Z_{d}$, which implies that $G /\left(G_{d+2} \Gamma\right)$ is also a $d$-step nilsystem. Now, by the maximality of $G /\left(G_{d+1} \Gamma\right)$, we have $G /\left(G_{d+2} \Gamma\right)$ and $G /\left(G_{d+1} \Gamma\right)$ coincide. Then, $G_{d+1} \Gamma=G_{d+2} \Gamma$, and of course the nilpotent Lie groups $G_{d+1}$ and $G_{d+2}$ have the same dimension since $\Gamma$ is discrete.

For any positive integer $k$, let $\mathfrak{g}_{k}$ be the associated Lie algebra of $G_{k}$. Then $\mathfrak{g}_{d+1}$ and $\mathfrak{g}_{d+2}$ have the same dimension, which implies that $\mathfrak{g}_{d+1}$ and $\mathfrak{g}_{d+2}$ coincide since $\mathfrak{g}_{d+2}$ is a subalgebra of $\mathfrak{g}_{d+1}$. Since $G_{d+1}$ and $G_{d+2}$ are connected, $G_{d+1}=$ $\exp \left(\mathfrak{g}_{d+1}\right)=\exp \left(\mathfrak{g}_{d+2}\right)=G_{d+2}$, and then we have that

$$
G_{d+3}=\left[G, G_{d+2}\right]=\left[G, G_{d+1}\right]=G_{d+2}=G_{d+1} .
$$

Inductively, we have $G_{k+1}=G_{d+1}$ for all $d \leq k \leq n$, which implies that $G_{d+1}=\{e\}$, $G$ is $d$-step nilpotent and $Z_{n, i}=G / \Gamma$ is a $d$-step nilsystem. So the inverse limit $Z_{n}$ is a $d$-step nilsystem. By the maximality of $Z_{d}$ we conclude $Z_{n}=Z_{d}$. The proof is completed. 
To show the next lemma we need some results from [16].

Proposition 8.2. Let $(X, T)$ be a minimal system. Then, If $(X, T)$ is an inverse limit of some d-step nilsystems, then each measurable factor is a topological factor.

Proof. It is a combination of [16, Proposition 5.2] and [16, Lemma 6.1].

Lemma 8.3. Let $(X, T)$ be a minimal system of order $n$, then the maximal measurable and topological factors of order $d$ coincide, where $d \leq n$.

Proof. Let $\mu$ be the unique invariant probability measure of $X$ and $Z_{d}$ is the maximal measurable factor of order $d$ of $(X, \mu, T)$. It is clear that $Z_{d}$ is a topological factor of order $d$ of $(X, \mu, T)$ by Proposition 8.2 . Endow the maximal topological factor $X_{d}$ of order $d$ of $X$ with its unique invariant probability measure. Clearly it is a measurable factor of order $d$ of $(X, \mu, T)$ and so is a measurable factor of $Z_{d}$. By Proposition 8.2 again, $X_{d}$ is a topological factor of $Z_{d}$, and so $Z_{d}=X_{d}$ is the maximal topological factor of order $d$.

Now we can finish the proof of Theorem 3.8 .

Proof of Theorem 3.8: (1) For any $k \geq 1$, recall $X_{k}=X / \mathbf{R P}^{[k]}$ and let $\mu_{k}$ be its unique invariant probability measure. Let $n>d$ be an integer. By the Lemma 8.3 and since $X_{n}$ is a minimal system of order $n$, then the maximal measurable and topological factors of order $k$ of $X_{n}$ coincide and $X_{n} / \mathbf{R P}^{[k]}\left(X_{n}\right)=X_{k}=Z_{k}$, $k \leq n$. As $\left(Z_{d}, \mu_{d}, T\right)=\left(Z_{d+1}, \mu_{d+1}, T\right)$, by Theorem $3.8(2)$, we have for any $d \leq$ $k \leq n,\left(Z_{k}, \mu_{k}, T\right)=\left(Z_{d}, \mu_{d}, T\right)$. Therefore $\left(Z_{n}, \mu_{n}, T\right)$ and $\left(Z_{d}, \mu_{d}, T\right)$ coincide in the measurable sense, and by Proposition 8.2 , they coincide in the topological sense too, i.e. $X_{n}=Z_{n}=Z_{d}=X_{d}$, which implies that $\mathbf{R P}^{[n]}(X)=\mathbf{R P}^{[d]}(X)$.

(3) If $Z_{n}$ is measure theoretical isomorphic with $X_{n}=X / \mathbf{R P}^{[n]}(X)$ for some $n \in \mathbb{N}$. By the Lemma 8.3. for any positive integer $k \leq n$, the measurable and topological maximal factors of order $k$ coincide, which implies $Z_{k}$ is measure theoretical isomorphic with $X_{k}=X / \mathbf{R P}^{[k]}(X)$. The proof of the theorem is completed.

\section{REFERENCES}

[1] J. Auslander, Minimal flows and their extensions, North-Holland Mathematics Studies, 153, (1988), North-Holland, Amsterdam.

[2] L. Auslander, L. Green and F. Hahn, Flows on homogeneous spaces, Annals of Mathematics Studies, 53, Princeton University Press, Princeton, N.J. 1963 vii+107 pp.

[3] V. Bergelson, B. Host and B. Kra, Multiple recurrence and nilsequences, Invent. Math., 160, (2005), 261-303, with an appendix by I.Z. Ruzsa.

[4] F. Blanchard, B. Host and A. Maass, Topological complexity, Ergodic Theory and Dynamical Systems, 20, (2000), 641-662.

[5] F. Blanchard and Y. Lacroix, Zero-entropy factors of topological flows, Proc. Amer. Math. Soc., 119, (1993), 985-992.

[6] H. Furstenberg, Strict ergodicity and transformation of the torus, Amer. J. Math., 83, (1961), 573-601. 
[7] H. Furstenberg, Recurrence in ergodic theory and combinatorial number theory, M. B. Porter Lectures, Princeton University Press, Princeton, N.J., 1981.

[8] E. Glasner, Proximal flows, Lecture Notes in Math. 517, Springer-Verlag, 1976.

[9] E. Glasner, Topological weak mixing and quasi-Bohr systems, Israel J. Math., 148, (2005), $277-304$.

[10] E. Glasner, The structure of tame minimal dynamical systems, Ergodic Theory and Dynamical Systems, 27, (2007), 1819-1837.

[11] E. Glasner and B. Weiss, Quasi-factors of zero-entropy systems, J. Amer. Math. Soc., 8, (1995), 665-686.

[12] E. Glasner and X. Ye, Local entropy theory, Ergodic Theory and Dynamical Systems, 29, (2009), 321-356.

[13] B. Green and T. Tao, The quantitative behaviour of polynomial orbits on nilmanifolds, to appear in Annals of Math.

[14] B. Host, Convergence of multiple ergodic averages, arXiv:0606362.

[15] B. Host and B. Kra, Nonconventional averages and nilmanifolds, Ann. of Math., 161, (2005) 398-488.

[16] B. Host, B. Kra and A. Maass, Nilsequences and a Structure Theory for Topological Dynamical Systems, Advances in Mathematics, 224, (2010) 103-129.

[17] W. Huang, Tame systems and scrambled pairs under an Abelian group action, Ergodic Theory and Dynamical Systems, 26, (2006), 1549-1567.

[18] W. Huang, H. Li and X. Ye, Family-independence for topological and measurable dynamics, arXiv: 0908.0574, to appear in Trans. Amer. Math. Soc..

[19] W. Huang, H. Li and X. Ye, Localization and dynamical Ramsey property, Preprint.

[20] W. Huang, S. Li, S. Shao and X. Ye, Null systems and sequence entropy pairs, Ergodic Theory and Dynamical Systems, 23, (2003), 1505-1523.

[21] W. Huang and X. Ye, Topological complexity, return times and weak disjointness, Ergodic Theory and Dynamical Systems, 24, (2004), 825-846.

$[22]$ W. Huang and X. Ye, A local variational relation and applications, Israel J. Math., 151, (2006), 237-280.

[23] B. Kra, Personal communication.

[24] D. Kerr and H. Li, Independence in topological and $C^{*}$-dynamics, Math. Ann., 338, (2007), 869-926.

[25] A. Leibman, Pointwise convergence of ergodic averages for polynomial sequences of translations on a nilmanifold, Ergodic Theory and Dynamical Systems, 25, (2005), no. 1, 201-213.

[26] E. Lindenstrauss, Measurable distal and topological distal systems, Ergodic Theory and Dynamical Systems, 19, (1999), no.4, 1063-1076.

[27] A. Maass and S. Shao, Sequence entropy in minimal systems, J. London of Math. Soc., 76, (2007), no. 3, 702-718.

[28] M. Pollicott, Fractals and Dimension Theory. Available at http://www.warwick.ac.uk/ masdbl/preprints.html

[29] S. Shao and X. Ye, Regionally proximal relation of order d is an equivalence one for minimal systems and a combinatorial consequence, arXiv:1007.0189.

[30] W. A. Veech, Point-distal flows, Amer. J. Math., 92, (1970), 205-242.

[31] W. A. Veech, Topological dynamics, Bull. Amer. Math. Soc., 83, (1977), 775-830.

[32] B. Weiss, Multiple recurrence and doubly minimal systems, Contemporary Math., 215, (1998), 189-196.

[33] J. van der Woude, Topological dynamics, Dissertation, Vrije Universiteit, Amsterdam, 1982. CWI Tract, 22. 
[34] T. Ziegler, Universal characteristic factors and Furstenberg averages. J. Amer. Math. Soc. 20 (2007), no. 1, 53-97.

[35] A. Zygmund, Trigonometric Series, second edition, University Press, Cambridge, 1959.

Wu Wen-Tsun Key Laboratory of Mathematics, UStC, Chinese Academy of Sciences and Department of Mathematics, University of Science and Technology of China, Hefei, Anhui, 230026, P.R. China.

E-mail address: dopandn@mail.ustc.edu.cn

Centro de Modelamiento Matemático and Departamento de Ingeniería Matemática, Universidad de Chile, Av. Blanco Encalada 2120, Santiago, Chile.

E-mail address: sdonoso@dim.uchile.cl

Centro de Modelamiento Matemático and Departamento de Ingeniería Matemática, Universidad de Chile, Av. Blanco Encalada 2120, Santiago, Chile.

E-mail address: amaass@dim.uchile.cl

Department of Mathematics, University of Science and Technology of China, Hefei, Anhui, 230026, P.R. China.

E-mail address: songshao@ustc.edu.cn

Department of Mathematics, University of Science and Technology of China, Hefei, Anhui, 230026, P.R. China.

E-mail address: yexd@ustc.edu.cn 